\title{
C90RF72 and RAB7L1 regulate vesicle trafficking in Amyotrophic Lateral Sclerosis and Frontotemporal Dementia
}

\begin{tabular}{|c|c|}
\hline Journal: & Brain \\
\hline Manuscript ID & BRAIN-2016-00318.R3 \\
\hline Manuscript Type: & Report \\
\hline Date Submitted by the Author: & 21-Dec-2016 \\
\hline Complete List of Authors: & $\begin{array}{l}\text { Aoki, Yoshitsugu; University of Oxford, Department of Physiology, Anatomy } \\
\text { and Genetics; Kokuritsu Seishin Shinkei Center, Department of Molecular } \\
\text { Therapy } \\
\text { Manzano, Raquel; University of Oxford, Department of Physiology, } \\
\text { Anatomy and Genetics } \\
\text { Lee, Yi; Karolinska Institutet, Department of Laboratory Medicine } \\
\text { Mutihac, Ruxandra; University of Oxford, Nuffield Department of Clinical } \\
\text { Neurosciences } \\
\text { Aoki, Misako; University of Oxford, Department of Physiology, Anatomy } \\
\text { and Genetics } \\
\text { Douglas, Andrew; University of Oxford, Department of Physiology, } \\
\text { Anatomy and Genetics } \\
\text { Varela, Miguel; University of Oxford, Department of Physiology, Anatomy } \\
\text { and Genetics } \\
\text { Sathyaprakash, Chaitra; University of Oxford, Nuffield Department of } \\
\text { Clinical Neurosciences } \\
\text { Scaber, Jakub; University of Oxford, Nuffield Department of Clinical } \\
\text { Neurosciences } \\
\text { Barbagallo, Paola; University of Oxford, Nuffield Department of Clinical } \\
\text { Neurosciences } \\
\text { Vader, Pieter; University of Oxford, Department of Physiology, Anatomy } \\
\text { and Genetics } \\
\text { Mäger, Imre; University of Oxford, Department of Physiology, Anatomy } \\
\text { and Genetics } \\
\text { Ezzat, Kariem; University of Oxford, Department of Physiology, Anatomy } \\
\text { and Genetics } \\
\text { Turner, Martin; University of Oxford, Nuffield Department of Clinical } \\
\text { Neurosciences } \\
\text { Ito, Naoki; Kokuritsu Seishin Shinkei Center, Department of Molecular } \\
\text { Therapy } \\
\text { Cavero, Samanta; University of Oxford, Department of Physiology, } \\
\text { Anatomy and Genetics } \\
\text { Ohbayashi, Norihiko; University of Tsukuba, Department of Physiological } \\
\text { Chemistry } \\
\text { El-Andaloussi, Samir; Karolinska Institutet, Department of Laboratory } \\
\text { Medicine } \\
\text { Takeda, Shin'ichi; Kokuritsu Seishin Shinkei Center, Department of } \\
\text { Molecular Therapy }\end{array}$ \\
\hline
\end{tabular}




\begin{tabular}{|c|c|}
\hline & $\begin{array}{l}\text { Fukuda, Mitsunori; Tohoku University, Department of Developmental } \\
\text { Biology and Neurosciences } \\
\text { Talbot, Kevin; University of Oxford, Nuffield Department of Clinical } \\
\text { Neurosciences } \\
\text { Wood, Matthew; University of Oxford, }\end{array}$ \\
\hline Subject category: & Neurodegeneration - cellular and molecular \\
\hline $\begin{array}{l}\text { To search keyword list, use } \\
\text { whole or part words followed } \\
\text { by an *: }\end{array}$ & $\begin{array}{l}\text { Frontotemporal dementia < DEMENTIA, Gene therapy < GENETICS, } \\
\text { Molecular genetics < GENETICS, Autophagy < NEURODEGENERATION: } \\
\text { CELLULAR AND MOLECULAR, Amyotrophic lateral sclerosis < } \\
\text { NEURODEGENERATION: CELLULAR AND MOLECULAR }\end{array}$ \\
\hline
\end{tabular}

\section{SCHOLARONE \\ Manuscripts}




\section{C9ORF72 and RAB7L1 regulate vesicle trafficking in Amyotrophic}

\section{Lateral Sclerosis and Frontotemporal Dementia}

Yoshitsugu Aoki ${ }^{1,5^{*}}$, Raquel Manzano ${ }^{1 *}$, Yi Lee ${ }^{1}$, Ruxandra Dafinca ${ }^{2}$, Misako Aoki ${ }^{1}$, Andrew G. L. Douglas ${ }^{1}$, Miguel A. Varela ${ }^{1}$, Chaitra Sathyaprakash ${ }^{2}$, Jakub Scaber ${ }^{2}$, Paola Barbagallo ${ }^{2}$, Pieter Vader ${ }^{1,3}$, Imre Mäger ${ }^{1}$, Kariem Ezzat ${ }^{1,4}$, Martin R. Turner ${ }^{2}$, Naoki Ito ${ }^{5}$, Samanta Gasco ${ }^{6}$ Norihiko Ohbayashi ${ }^{7}$, Samir El Andaloussi ${ }^{1,4}$, Shin'ichi Taked $^{5}$, Mitsunori Fukuda ${ }^{8}$, Kevin Talbot ${ }^{2}$, Matthew J.A. Wood ${ }^{1}$

1 Department of Physiology, Anatomy and Genetics, University of Oxford, South Parks Road, Oxford, OX1 3QX, United Kingdom.

2 Nuffield Department of Clinical Neurosciences, University of Oxford, Level 6, West Wing, John Radcliffe Hospital, Oxford OX3 9DU, United Kingdom.

3 Department of Clinical Chemistry and Haematology, University Medical Center Utrecht, Utrecht, the Netherlands.

4 Department of Laboratory Medicine, Karolinska Institutet, Huddinge, Sweden.

5 Department of Molecular Therapy, National Institute of Neuroscience, National Center of Neurology and Psychiatry (NCNP), Kodaira, Tokyo, 187-8502, Japan.

6 Lagenbio, Veterinary Faculty of Zaragoza, Instituto Agroalimentario de Aragon (I2A), Health Research Institute of Aragon (IIS), University of Zaragoza, Zaragoza, Spain. 
7 Department of Physiological Chemistry, Faculty of Medicine and Graduate School of Comprehensive Human Sciences, University of Tsukuba, Tsukuba, Ibaraki 305-8575, Japan.

8 Department of Developmental Biology and Neurosciences, Graduate School of Life Sciences, Tohoku University, Sendai, Miyagi 980-8578, Japan.

* These authors contributed equally to this work To whom correspondence should be addressed: Matthew J.A. Wood and Yoshitsugu Aoki yoshitsugu.aoki@dpag.ox.ac.uk and matthew.wood@dpag.ox.ac.uk Running title: C9ORF72 regulates vesicular trafficking

Keywords: C9ALS/FTD, C9ORF72, RAB7L1, extracellular vesicles, haploinsufficiency

Abbreviations: iPSC; induced pluripotent stem cells, ALS; amyotrophic lateral sclerosis, FTD; frontotemporal dementia, EV; extracellular vesicle, MVEs; multivesicular endosomes, NTA; nanoparticle tracking analysis, HRE; hexanucleotide repeat expansion, M6PR; mannose-6-phosphate receptor, EBSS; Earle's Balanced Salt Solution, WB; western blotting 


\section{ABSTRACT}

A non-coding hexanucleotide repeat expansion (HRE) in intron 1 of the C9ORF72 gene is the most common cause of amyotrophic lateral sclerosis and frontotemporal dementia (C9ALS/FTD), however, the precise molecular mechanism by which the C9ORF72 HRE directs C9ALS/FTD pathogenesis remains unclear. Here, we report a novel disease mechanism arising due to the interaction of C9ORF72 with the RAB7L1 GTPase to regulate vesicle trafficking. Endogenous interaction between C9ORF72 and RAB7L1 was confirmed in human SH-SY5Y neuroblastoma cells. The C9ORF72 HRE led to haploinsufficiency resulting in severely defective intracellular and extracellular vesicle trafficking and a dysfunctional trans-Golgi network phenotype in patient-derived fibroblasts and iPSC-derived motor neurons. Genetic ablation of RAB7L1or C9ORF72 in SH-SY5Y cells recapitulated the findings in C9ALS/FTD fibroblasts and iPSCneurons. When C9ORF72 was overexpressed or antisense oligonucleotides were targeted to the C9ORF72 HRE to upregulate normal variant 1 transcript levels, the defective vesicle trafficking and dysfunctional trans-Golgi network phenotypes were reversed, suggesting that both loss- and gain-of-function mechanisms play a role in disease pathogenesis. In conclusion, we have identified a novel mechanism for C9ALS/FTD pathogenesis highlighting the molecular regulation of intracellular and extracellular vesicle trafficking as an important pathway in C9ALS/FTD pathogenesis.

\section{Introduction}

Amyotrophic lateral sclerosis (ALS) is the most common adult-onset motor neuron degenerative disease. Frontotemporal dementia (FTD) is characterised by degeneration of the frontal and temporal lobes, resulting in altered personality, behaviour and language. A hexanucleotide repeat expansion (HRE) in the chromosome 9 open reading 
frame $72(C 9 O R F 72)$ gene is the commonest single genetic cause of ALS/FTD, which constitutes a spectrum disorder in which both phenotypes overlap (Renton et al., 2011), but the mechanism by which C9ORF72 HRE causes neurotoxicity is unknown. Bioinformatic analysis has predicted that C9ORF72 contains a 'differentially expressed in normal and neoplasia' (DENN)-like domain, which can function as a GDP-GTP exchange factor for RAB-GTPases such as RAB7L1 and may therefore regulate endosomal trafficking (Levine et al., 2013)(Farg et al., 2014). Human RAB7L1 localizes primarily to the Golgi apparatus and its role in vesicular trafficking has been demonstrated previously, including its role in extracellular vesicle (EV) secretion (Shimizu et al., 1997)(Wang et al., 2014)(Berger et al., 2009)(Spanò et al., 2011).

Exosomes are small subclass of EVs, around 40-100 $\mathrm{nm}$ in diameter, that convey important biological information between cells in both normal physiological and pathological situations. They originate from the endosomal pathway, and are released/secreted from cells when multivesicular endosomes (MVEs) fuse with the plasma membrane (EL Andaloussi et al., 2013). Interestingly, in certain cell types, formation of MVEs and subsequent fusion events are driven by RAB GTPases and their effector proteins (Ostrowski et al., 2010). Moreover, increasing evidence suggests that compromised secretion of such EVs may contribute significantly to neurodegenerative disorders (Takeuchi et al., 2015)(Gilthorpe et al., 2013).

In this study, we investigated whether the C9ORF72 mutation disrupted RAB7L1mediated trans-Golgi network trafficking and EV secretion and to what extent and how this contributed to the pathogenesis of C9ALS/FTD. To address this, we analysed vesicle trafficking in human iPSC-derived motor neurons, fibroblasts and SH-SY5Y cell lines with altered expression of $C 9 O R F 72$ and $R A B 7 L 1$. We identified disruptions of 
both intracellular and extracellular vesicular trafficking that underlie a novel link between EV biogenesis and ALS/FTD pathogenesis.

\section{Materials and methods}

\section{Generation and culture of iPSC lines}

All iPSC lines were derived from skin biopsy fibroblasts in the James Martin Stem Cell Facility, University of Oxford, using standardized protocols. Biopsies were from patients attending the Oxford Motor Neuron Disease clinic and taken under ethical approval granted by the South East Wales Research Ethics Committee (Ref No, 12/WA/0186). Sendai-transduced fibroblasts were seeded onto a layer of mitomycin Cinactivated mouse embryonic feeder (MEF) cells on $0.1 \%$ gelatine-coated plates. MEFs were prepared from outbred Swiss mice (Department of Pathology, Oxford) and were cultured in iPS medium consisting of knock-out Dulbecco's Modified Eagle Medium (DMEM) (Invitrogen, CA), 10\% knock-out serum replacement (Invitrogen), $2 \mathrm{mM}$ Glutamax-I (Gibco), $100 \mathrm{U} / \mathrm{mL}$ penicillin (Invitrogen), $100 \mu \mathrm{g} / \mathrm{mL}$ streptomycin (Invitrogen), 1\% non-essential amino acids (Invitrogen), $0.5 \mathrm{mM} \beta$-mercaptoethanol (Invitrogen) and $10 \mathrm{ng} / \mathrm{mL}$ bFGF (R\&D). Half of the medium was replaced daily and substituted with MEF-conditioned medium from day 10 onwards. Colonies with iPSC morphology were picked manually on days 21-28 and transferred onto MEF layers every 5-7 days to increase stocks. Prior to differentiation, iPSC lines were adapted to MEF-free conditions on Matrigel-coated plates (BD Matrigel hESC-qualified Matrix) in mTeSR ${ }^{\mathrm{TM}} 1$ (StemCell Technologies) supplemented with Rock inhibitor Y27632 (10 $\mu \mathrm{M}$; Calbiochem) on the day of passage. The number of MEF-free passages was limited to ensure that differentiation experiments were all performed within a narrow window of passage numbers. 


\section{Differentiation of iPSCs to motor neurons}

Motor neurons were differentiated from iPSCs using a previously published protocol, with extensive modifications, as described in a separate manuscript (Hu and Zhang, 2009). Briefly, the differentiation process was initiated by neural induction of embryoid bodies (EBs) which were plated onto Geltrex-coated plates four days later in DMEM:F12 supplemented with N2 and B27 (Life Technologies). Caudalization of the neural progenitors was obtained by addition of $0.1 \mu \mathrm{M}$ retinoic acid (RA) to the neural differentiation medium (Sigma-Aldrich). After 10 days, the colonies displaying neural rosettes structures were isolated and expanded in suspension in the form of neurospheres in the presence of the ventralizing growth factor, sonic hedgehog (SHH) at a concentration of $100 \mathrm{ng} / \mathrm{mL}$ (PeproTech). To promote cell survival, $10 \mu \mathrm{M}$ Rock inhibitor Y27632 was added to the culture medium. After two weeks, the neurospheres were plated on Geltrex coated coverslips and final differentiation was induced by supplementing the medium with brain derived neurotrophic factor (BDNF $10 \mathrm{ng} / \mathrm{mL}$ ), glial-derived neurotrophic factor (GDNF, $10 \mathrm{ng} / \mathrm{mL}$ ), insulin-like growth factor (IGF-1, $10 \mathrm{ng} / \mathrm{mL}$ ), cyclic AMP (cAMP) and ascorbic acid (Life Technologies). The MNs were cultured for another 3-4 weeks to reach maturity before functional assays were performed.

\section{Culture of COS7 cells, SH-SY5Y cells and primary human fibroblasts}

Primary fibroblasts and COS7 cell lines were grown in DMEM supplemented with 10\% (vol/vol) foetal bovine serum (FBS) and 1\% (vol/vol) penicillin/streptomycin. The human SH-SY5Y neuroblastoma cell line was maintained in DMEM/F-12 1:1 (Invitrogen, San Diego, CA, USA) medium supplemented with 10\% (vol/vol) FBS and 
$1 \%(\mathrm{vol} / \mathrm{vol})$ penicillin/streptomycin. For starvation experiments, cells were grown in serum- and amino acid-depleted EBSS medium (Sigma) or Opti-MEM serum-free media (Gibco, Life Technologies) for the times indicated. All cells were maintained in a $37^{\circ} \mathrm{C}$ incubator with $5 \% \mathrm{CO}_{2}$.

\section{GST pull-down assay}

A pEF/Myc tag expression vector containing C9ORF72 (4 $\mu \mathrm{g}$ plasmid DNA) was transfected into COS-7 cells $\left(3 \times 10^{5}\right.$ cells $/ 6 \mathrm{~cm}$ dish, one day before transfection $)$ using Lipofectamine Plus reagent (Invitrogen) according to the manufacturer's protocol. Three days after transfection, cells were harvested and homogenized and total cell lysates were prepared as described previously (Kanno et al., 2010). Glutathionesepharose beads (10 $\mu \mathrm{L}$ wet volume) coupled with approximately $5 \mu$ g purified GSTRAB-GTPases were incubated for 20 min at $4{ }^{\circ} \mathrm{C}$ with $50 \mathrm{mM}$ HEPES-KOH, pH 7.2, $150 \mathrm{mM} \mathrm{NaCl}$ and $2.5 \mathrm{mM}$ EGTA.MgCl 2 and $\mathrm{GTP} \gamma \mathrm{S}$ were added to the solution at a final concentration of $10 \mathrm{mM}$ and $0.5 \mathrm{mM}$, respectively. The GST-RAB-GTPase beads were incubated for $1 \mathrm{~h}$ at $4{ }^{\circ} \mathrm{C}$ with $400 \mu \mathrm{L}$ cell lysate in $50 \mathrm{mM}$ HEPES-KOH, pH 7.2, $150 \mathrm{mM} \mathrm{NaCl}, 1 \mathrm{mM} \mathrm{MgCl} 2,1 \%$ Triton $\mathrm{X}-100$ and protease inhibitors in $0.5 \mathrm{mM}$ GTP $\gamma \mathrm{S}$ (or $1 \mathrm{mM}$ GDP). After washing the beads three times with $1 \mathrm{~mL} 50 \mathrm{mM}$ HEPES-KOH, pH 7.2, $150 \mathrm{mM} \mathrm{NaCl}, 1 \mathrm{mM} \mathrm{MgCl} 2,0.2 \%$ Triton X-100 and protease inhibitors, proteins bound to the beads were analysed by $10 \%$ SDS-PAGE followed by immunoblotting, using an anti-Myc-tagged antibody and a horseradish peroxidaseconjugated anti-GST antibody (Santa Cruz Biotechnology, Inc.). The immunoreactive bands were visualized using enhanced chemiluminescence (GE Healthcare Ltd.). Blots shown in the figures are representative of at least three independent experiments.

\section{Purification of EV}


EVs were purified by ultracentrifugation (UC) or ultrafiltration with size-exclusion liquid chromatography (UF-LC) as previously described (Nordin et al., 2015). Briefly, cell-conditioned media was centrifuged at $300 \mathrm{~g}$ for $5 \mathrm{~min}$, then at 1,200 $\mathrm{g}$ for $10 \mathrm{~min}$. The supernatant was passed through a $0.22 \mu \mathrm{m}$ filter then analysed by nanoparticle tracking analysis (NTA). For western blotting (WB), fluorescence-activated cell-sorting (FACS) and transmission electron microscopy, EVs were purified by a subsequent UC high-speed spin (at 120,000 $\mathrm{g}$ for $70 \mathrm{~min}$ ) or UF-LC.

\section{Nanoparticle tracking analysis (NTA)}

For particle-size determination, NTA was performed with a NanoSight NS500 instrument (Malvern) equipped with NTA 2.3 analytical software. For all recordings, we used a camera level of 13 or 15 and automatic function for all post-acquisition settings. Blur and minimum expected particle size, except for the detection threshold, were fixed at 5. Samples were thawed on ice and diluted between 1:10-1:500 in PBS to achieve a particle count of $2 \times 10^{8}-1 \times 10^{9}$ per $\mathrm{mL}$. The diluted sample was loaded in the sample chamber and the camera focus was adjusted so the particles appeared as focused dots. Using the script control function, we recorded three $30 \mathrm{~s}$ videos for each sample, incorporating a sample advance and $5 \mathrm{~s}$ delay between each recording. These measurements were analysed using the batch process function and results were analysed using Microsoft Excel.

\section{Statistical analysis.}

Two sample comparisons were tested for statistical significance using a two-tailed Student's t test. More than two groups were compared by one or two-way analysis of variance (ANOVA) and post-hoc Bonferroni or Dunnett's test correction where corresponded. $P$ values $<0.05$ were considered statistically significant. 


\section{Results}

\section{Reduced EV secretion in C9ALS/FTD fibroblasts and iPSC-derived motor neurons}

Nanoparticle tracking analysis (NTA) of conditioned serum-free medium from 4 different C9ALS/FTD patient fibroblasts (C9-1, C9-2, C9-3 and C9-4) (Table S1) identified the presence of EVs approximately $80-100 \mathrm{~nm}$ in diameter, corresponding to that reported for exosomes. Importantly, there were significantly fewer EVs as compared with healthy fibroblasts after 16 hours of incubation (Fig. 1a, b, S1a). EV secretion by C9ALS/FTD patient fibroblasts was also reduced in serum-free medium and amino acid-free EBSS buffer compared with normal EV-depleted medium at a shorter incubation period of 4 hours (data not shown). Unexpectedly, however, at longer incubation time points (48 hours), there were no differences in EV secretion by C9ALS/FTD patient fibroblasts and normal fibroblasts cultured in conditioned serumfree medium, evaluated by WB analysis of exosomal marker expression (TSG101, ALIX and CD81) (Fig. S1b). These data suggest that C9ORF72 mutations impair EV secretion when cells are under stress (such as serum-deprivation), but compensatory mechanisms may arise over time in vitro to offset this impairment.

Moreover, based on LC fractionation of cell secretome/conditioned media, we observed greater amounts of soluble protein secreted from C9-3 fibroblasts as compared to normal controls (Fig. S1c), indicating the possibility of disrupted protein trafficking between the endoplasmic reticulum and Golgi network (Nordin et al., 2015)(Lee et al., 2004). Even though EV release was greatly reduced in diseased fibroblasts, exosomal markers CD9 and CD63 in EVs secreted by C9ALS/FTD fibroblasts were detected using flow cytometry and immunocapture beads (Fig. 1c). To investigate intracellular processes that lead to defective EV secretion in C9ALS/FTD cells, we examined 
expression of the early endosome antigen 1 marker (EEA1) and the MVE/late endosome marker CD63 in C9ALS/FTD and normal fibroblasts. As expected, CD63 and EEA1 partially co-localized in fibroblasts from normal individuals and C9ALS/FTD patients (Fig. 1d, S2). Next, we investigated extracellular vesicle trafficking in normal and C9ALS/FTD fibroblasts. Interestingly the number of CD63-positive granules observed by immunofluorescence was significantly lower in C9ALS/FTD fibroblasts (Fig. 1d and e, S2), indicating a lower number of MVEs in C9ALS/FTD fibroblasts and significantly reduced EV secretion (Fader et al., 2008)

Motor neuron degeneration is a cardinal feature of C9ALS/FTD, therefore we also examined EV secretion in motor neurons differentiated from C9ALS/FTD iPSCs (Dafinca et al., 2016). We observed a reduced EV secretion in C9ALS/FTD motor neurons by NTA (Fig. 1f).

\section{C9ORF72 interacts with RAB7L1 to mediate trans-Golgi vesicle trafficking}

To determine the mechanism underlying the effect of the C9ORF72 mutation on EV secretion, we examined the role of the C9ORF72 protein in cellular vesicle trafficking. C9ORF72 contains a DENN-like domain, which may serve as a GDP-GTP exchange factor for RAB GTPases. We hypothesized that C9ORF72 regulates endosomal trafficking by interacting with certain RAB-GTPases, based on our findings that C9ALS/FTD fibroblasts have impaired EV secretion. To test which RAB family members bind C9ORF72, we performed GST pull-down assays using 42 different mammalian RAB proteins and myc-tagged forms of human C9ORF72 (Kanno et al., 2010). Unexpectedly, we observed highly specific bands indicating an interaction between C9ORF72 and the recently identified RAB7L1 (MacLeod et al., 2013) (Fig. 2a, S3a). We did not observe a significant interaction between C9ORF72 and more 
well-known RAB proteins, such as RAB42 (RAB7B), RAB15 and RAB10. We postulate that this result might be due to differential expression of such RAB proteins in our C9ALS/FTD iPSC-motor neurons and fibroblasts; high expression of RAB7L1 and lower expression of RAB15, RAB42 and RAB10 (Fig. S3b). Therefore, we focused our further investigations on unravelling the link between the C9ORF72-RAB7L1 interaction and endosomal trafficking.

Immunofluorescence with the Golgi marker GM130 showed that C9ORF72 is enriched in the Golgi apparatus of C9ALS/FTD and normal fibroblasts (Fig. S4a). Interestingly, RAB7L1 has been also described as enriched in the Golgi apparatus (MacLeod et al., 2013). Unfortunately, antibodies for RAB7L1 immunofluorescence failed to detect endogenous levels of RAB7L1 using this technique. To test the interaction between C9ORF72 and RAB7L1, C9ALS/FTD patient fibroblasts were transfected with either a constitutively active (CA) RAB7L1 mutant (Q67L) or a constitutively negative (CN) RAB7L1 mutant (T21N) (Fig. 2b). Based on this immunofluorescence staining, we observed co-localization of C9ORF72 with the constitutively active RAB7L1 mutant resulting in a vesicular staining pattern of both proteins. Constitutively negative $(\mathrm{CN})$ RAB7L1 mutant (T21N) appeared, as previously reported, in a diffuse pattern, and so did C9ORF72, which suggests certain degree of interaction between C9ORF72 and CN RAB7L1 as well. It is reported that most of the GDP-bound CN forms of RAB proteins prevent recruitment of downstream effectors maybe via a GDP/GTP exchange factortrap mechanism (Mori et al., 2013) and therefore are diffusely localized in the cytosol. By contrast, the GTP-bound CA forms are often enriched in punctate structures, specifically targeted vesicles or other intracellular compartments (Zhang et al., 2007), presumably because the CA proteins lack GTPase activity and stably present at transport vesicles/organelles. 
Next, the endogenous interaction between C9ORF72 and RAB7L1 was shown in SHSY5Y neuroblastoma cells by a proximity ligation assay using Duolink PLA probes (Fig. 2c). Finally, we confirmed that C9ORF72 protein interacted strongly and preferentially with the constitutively active mutant form of RAB7L1 (which mimics the GTP-bound form) in a co-immunoprecipitation assay (Fig. 2d). All these data suggest that C9ORF72 is likely to function as a RAB7L1 effector (Fukuda et al., 2008). Our findings here indicate that C9ORF72 and RAB7L1 interact and are enriched in the Golgi apparatus of fibroblasts. However, in C9ALS/FTD iPSC-motor neurons where the C9ORF72 protein is downregulated due to the HRE mutations (Fig. 2e, f), we hypothesized that this C9ORF72-RAB7L1 interaction is disrupted, and consequently results in defective trans-Golgi network trafficking.

To further characterize the dysfunction of trans-Golgi network trafficking, we investigated the localization of the mannose-6-phosphate receptor (M6PR) which transports hydrolase precursors from the trans-Golgi network to late endosomes as they are recycled to the trans-Golgi network via the heteropentameric retromer complex comprising VPS26, VPS29 and VPS35 (Arighi et al., 2004). Interestingly, M6PR was expressed throughout the cytoplasm in C9ALS/FTD fibroblasts, whereas in normal fibroblasts M6PR was detected mainly around the nucleus (Fig. 2g, h, S4b), suggesting an impaired recycling function in C9ALS/FTD cells. In addition, the retromer VPS26 protein level showed a tendency to be decreased in C9ALS/FTD cells (Fig. 2e, f) while mRNA expression of VPS26, VPS29 and VPS35 retromer units, and M6PR was upregulated in C9ALS/FTD iPSC-motor neurons (Fig. S5). These mRNA findings might represent a compensatory mechanism for defective intracellular M6PR sorting between late endosomes and the trans-Golgi network. Collectively, these data demonstrate a direct interaction between C9ORF72 and RAB7L1 in human neurons and 
fibroblasts that is disrupted by C9ORF72 protein loss, probably due to the HRE mutation in cells carrying the $C 9 O R F 72$ mutation, resulting in compromised trans-Golgi network vesicle trafficking.

Dysfunction of the trans-Golgi network inhibits autophagy and lysosomal degradation pathways (Eskelinen and Saftig, 2009). Recently, it was shown that autophagy is compromised in C9ORF72 deficient neurons and in iPSC-motor neurons from C9ALS/FTD patients (Stepto et al., 2014). Here, we confirmed increased expression of the autophagosome markers p62 and LC3-II in C9ALS/FTD patient-derived fibroblasts (Fig. S6a, b), in p62-positive autophagosomes (Fig. S6d, e) and swollen autophagosomes in iPSC-motor neurons as determined by transmission electron microscopy (Fig. S6c), suggesting impaired degradation of autophagosomes by lysosomal enzymes. Disruption in the trans-Golgi network and leucine-rich repeat kinase 2 (LRRK2) can inhibit autophagy over time, in the absence of compensatory mechanisms (MacLeod et al., 2013). In our case, amino acid-free starvation-induced stress reduced the number of p62-positive autophagosomes (Fig. S6d, e) and p62 protein levels in C9ALS/FTD fibroblasts compared with normal fibroblasts, even after suppression of lysosomal degradation by E64d and pepstatin (Fig. S7a, b). Hence, our findings indicate inhibition of autophagy, in particular in C9ALS/FTD patient fibroblasts under stress conditions.

\section{Genetic ablation of $R A B 7 L 1$ and $C 9 O R F 72$ by siRNA in SH-SY5Y cells recapitulates the C9ALS/FTD phenotype.}

To further examine the haploinsufficiency hypothesis we investigated whether genetic ablation of C9ORF72 and RAB7L1 in SH-SY5Y cells was sufficient to induce the C9ALS/FTD vesicle trafficking phenotype. We achieved a 70\% knockdown of C9ORF72 and an 85\% knockdown of RAB7L1 in SH-SY5Y cells using short interfering 
RNA (siRNA) (Fig. S8). C9ORF72 and RAB7L1 knockdown recapitulated the EV secretion phenotype observed in C9ALS/FTD fibroblasts, as determined by NTA (Fig. 3a) and WB of the exosomal markers ALIX and TSG101 (Fig. 3b). Moreover, RAB7L1 or C9ORF72 knockdown resulted in reduced expression of the EV markers CD9, CD63 and CD81 in purified EV samples from starved SH-SY5Y cells (Figs. 3c, d, e). Silencing $C 9 O R F 72$ and $R A B 7 L 1$ reduced p62 expression after amino acid-starvation (Fig. S7c), while overexpression of both C9ORF72 and RAB7L1 significantly upregulated MVE and EV secretion (Fig. 3f, g, h, S9) in C9ALS/FTD fibroblasts. These data demonstrate that defects in the C9ORF72-RAB7L1 pathway significantly reduce MVE production, EV secretion and autophagy in amino acid-depleted conditions, similar to our findings in C9ORF72 HRE patient derived cells.

Here, we demonstrate that fibroblasts and motor neurons derived from ALS/FTD patients have impaired function in endosomal trafficking, regulation of trans-Golgi network trafficking and autophagy. These effects are further pronounced under stress conditions such as amino acid depletion and could accumulate over time to result in neurodegeneration (Zhang et al., 2015).

Finally, we evaluated the therapeutic potential of a novel short locked nucleic acid (LNA)-DNA-LNA oligonucleotide gapmer against C9ALS/FTD (Fig. S10a, b, c and Table S2). The LNA gapmer interferes with C9ORF72 pre-mRNA processing in C9ALS/FTD patient fibroblasts and we wished to investigate if it could reverse the RAB7L1-related disease phenotype. In addition, it targets the region immediately upstream of the GGGGCC repeat in intron 1a (ASO2-1) and significantly upregulates the main C9ORF72 1b transcript (Fig. 4a, b) and C9ORF72 protein levels (Fig. 4c, d) 4 days after specific knockdown of the mutant C9ORF72 transcripts. Reduced EV secretion (Fig. S11a) and mislocalization of M6PR to the cytoplasm (Fig. S11b, c) were 
rescued by knockdown of the mutant C9ORF72 transcripts in C9ALS/FTD patient fibroblasts using this LNA gapmer. These findings highlight the therapeutic potential for ALS/FTD of using this oligonucleotide approach.

\section{Discussion}

The mechanisms underlying the neurotoxicity caused by the C9ORF72 HRE mutation have not been fully elucidated, although RNA gain-of-function, polydipeptide toxicity caused by repeat-associated non-ATG translation and loss of gene function have all been suggested (Aoki et al., 2015). Abnormal endocytic trafficking has been reported in several neurodegenerative diseases, including ALS (Jovic et al., 2015), Alzheimer's disease, Down syndrome and Parkinson's disease (Wang et al., 2014)(Muresan and Ladescu Muresan, 2015). In this study, we revealed a novel interaction between C9ORF72 and RAB7L1, which is disrupted by downregulation of the C9ORF72 protein in the presence of the HRE mutation. Inhibition of the C9orf72 HRE transcript with ASO2-1, suggests that this downregulation of the main C9ORF72 $1 \mathrm{~b}$ transcript, leading to C9ORF72 protein loss, appears to be reversible. Also, normalization of the Golgi phenotype after ASO treatment indicates that this aspect of the C9/ALS pathogenesis is, at least partially, related to a direct effect of the HRE transcripts.

The consequence of this disrupted interaction is dysfunctional trans-Golgi network trafficking and compromised MVE formation, resulting in defective intracellular and extracellular vesicle trafficking which represents a possible pathogenic mechanism in ALS/FTD (Fig. 4e). How impaired vesicular trafficking leads to late-onset neurodegenerative diseases such as C9ALS/FTD remains to be determined. Evidence suggests that an accumulation of age-dependant effects on an appropriate genetic background can predispose an individual to diseases like ALS (Al-Chalabi et al., 2014). Very recently, it has been reported that C9ORF72 complex acts as a GDP/GTP 
exchange factor for RAB8a and RAB39b, and it synergizes with Ataxin-2 Q30x toxicity to induce motor neuron dysfunction and neuronal cell death (Sellier et al., 2016). In keeping with other genetic forms of ALS/FTD, the presence of the C9ORF72 mutation is not a sufficient condition for neurodegeneration, but only precipitates neurodegeneration after many years have elapsed, with aging as a co-factor.

In the central nervous system, RAB7L1 interacts with LRRK2 to regulate intra-neuronal protein sorting and disruptions in his process are a risk factor for Parkinson's disease (MacLeod et al., 2013). EVs and autophagy are important for neuronal maintenance and survival; therefore, reduced EV secretion and impaired autophagy could render neurons more vulnerable to further neurodegenerative insults over time. Our data strongly suggest that C9ORF72 haploinsufficiency directly mediated by the HRE, which is known to induce hypermethylation, are major contributing factors to ALS/FTD spectrum disorders, in agreement with previous findings (Haeusler et al., 2014)(Jovic et al., 2015)(Zhang et al., 2015).

Due to the lack of appropriate animal models carrying the C9ORF72 HRE, a limitation of our study is that we did not examine EV biogenesis in vivo. Future work to measure EV secretion in the cerebrospinal fluid of C9ALS/FTD patients could help clarify the relevance of our findings to the clinical disease. The development of models reproducing C9ALS/FTD pathology will enable us to investigate the long-term effects of the C9ORF72-RAB7L1 interaction and its influence on EV biogenesis as well as the therapeutic potential of LNA gapmer-based interventions for the disease.

In conclusion, we have identified an association between C9ORF72 mutations and the RAB7L1-based regulation of vesicular trafficking, indicating a common potential pathogenic mechanism that can be rescued by antisense-based intervention. This work 
may pave the way to the development of novel therapies for ALS and FTD that target the C9ORF72-RAB7L1 pathway.

\section{Acknowledgements}

The authors thank Dr. Shih-Jung Fan, Dr. Deborah Goberdhan, Dr. Clive Wilson, Dr. Kenji Hyodo, Dr. Errin Johnson and Dr. Yuko Hara for scientific advice and technical assistance. We are grateful to Dr. Sally Cowley and the Stem Cell Facility at Oxford University for the reprogramming of fibroblasts.

\section{Funding}

The work was supported by the Uehara Memorial Foundation (grant to Y.A.), the MRC Confidence in Concept award (grant to Y.A. and K.T.), the John Fell Fund award (grant to Y.A. and K.T.) and a VENI fellowship from the Netherlands Organization for Scientific Research (NWO) (to P.V.). S.EL.A. is supported by the Swedish Research council (VR-Med and EuroNanomed) and the Swedish Society of Medical Research (SSMF).

\section{Conflicts of interest}

The authors declare no competing financial interests.

\section{Supplementary material}

Supplementary material is available at Brain online.

\section{References}

Al-Chalabi A, Calvo A, Chio A, Colville S, Ellis CM, Hardiman O, et al. Analysis of amyotrophic lateral sclerosis as a multistep process: a population-based modelling study [Internet]. Lancet Neurol. 2014; 13: 1108-1113.Available from: http://linkinghub.elsevier.com/retrieve/pii/S1474442214702194

Almeida S, Gascon E, Tran H, Chou HJ, Gendron TF, Degroot S, et al. Modeling key pathological features of frontotemporal dementia with C9ORF72 repeat expansion in 
iPSC-derived human neurons. [Internet]. Acta Neuropathol. 2013; 126: 385-99.[cited 2014 Feb 25] Available from: http://www.pubmedcentral.nih.gov/articlerender.fcgi? artid=3753484\&tool=pmcentrez\& $\underline{\text { rendertype }=\text { abstract }}$

EL Andaloussi S, Mäger I, Breakefield XO, Wood MJ a. Extracellular vesicles: biology and emerging therapeutic opportunities. [Internet]. Nat. Rev. Drug Discov. 2013; 12: 347-57.[cited 2014 Jul 10$]$ Available from: http://www.ncbi.nlm.nih.gov/pubmed/23584393

Aoki Y, Douglas AGL, Wood MJA. Oligonucleotide therapies: the future of amyotrophic lateral sclerosis treatment ? 2015; 5: 93-95.

Arighi CN, Hartnell LM, Aguilar RC, Haft CR, Bonifacino JS. Role of the mammalian retromer in sorting of the cation-independent mannose 6-phosphate receptor. [Internet]. J. Cell Biol. 2004; 165: 123-33. [cited 2015 Feb 8] Available from: http://www.pubmedcentral.nih.gov/articlerender.fcgi?artid=2172094\&tool=pmcentrez\& rendertype $=$ abstract

Berger KL, Cooper JD, Heaton NS, Yoon R, Oakland TE, Jordan TX, et al. Roles for endocytic trafficking and phosphatidylinositol 4-kinase III alpha in hepatitis $\mathrm{C}$ virus replication. Proc. Natl. Acad. Sci. U. S. A. 2009; 106: 7577-7582.Dafinca R, Scaber J, Ababneh N, Lalic T, Weir G, Christian H, et al. C9orf72 Hexanucleotide Expansions Are Associated with Altered Endoplasmic Reticulum Calcium Homeostasis and Stress Granule Formation in Induced Pluripotent Stem Cell-Derived Neurons from Patients with Amyotrophic Lateral Sclerosis and Frontotemporal Dementia. Stem Cells. 2016;34:2063-78.

Dafinca R, Scaber J, Ababneh N, Lalic T, Weir G, Christian H, et al. C9orf72 Hexanucleotide Expansions Are Associated with Altered Endoplasmic Reticulum Calcium Homeostasis and Stress Granule Formation in Induced Pluripotent Stem CellDerived Neurons from Patients with Amyotrophic Lateral Sclerosis and Frontotemporal Dementia. Stem Cells. 2016 ;34:2063-78.

Donnelly CJ, Zhang P-W, Pham JT, Heusler AR, Mistry N a, Vidensky S, et al. RNA toxicity from the ALS/FTD C9ORF72 expansion is mitigated by antisense intervention. [Internet]. Neuron 2013; 80: 415-28.Available from: http://www.ncbi.nlm.nih.gov/pubmed/24139042

Eskelinen E-L, Saftig P. Autophagy: A lysosomal degradation pathway with a central role in health and disease. Biochim. Biophys. Acta - Mol. Cell Res. 2009; 1793: 664673.

Fader CM, Sánchez D, Furlán M, Colombo MI. Induction of autophagy promotes fusion of multivesicular bodies with autophagic vacuoles in k562 cells. [Internet]. Traffic 2008; 9: 230-50.[cited 2014 Feb 25] Available from: http://www.ncbi.nlm.nih.gov/pubmed/17999726

Farg M a, Sundaramoorthy V, Sultana JM, Yang S, Atkinson R a K, Levina V, et al. C9ORF72, implicated in amytrophic lateral sclerosis and frontotemporal dementia, regulates endosomal trafficking. [Internet]. Hum. Mol. Genet. 2014; 23: 3579-95.[cited 2014 Jul 14] Available from: http://www.pubmedcentral.nih.gov/articlerender.fcgi?artid=4049310\&tool=pmcentrez\& $\underline{\text { rendertype }=\text { abstract }}$ 
Fukuda M, Kanno E, Ishibashi K, Itoh T. Large scale screening for novel rab effectors reveals unexpected broad Rab binding specificity. [Internet]. Mol. Cell. Proteomics 2008; 7: 1031-1042.Available from: http://www.mcponline.org/content/7/6/1031.long

Gilthorpe JD, Oozeer F, Nash J, Calvo M, Bennett DL, Lumsden A, et al. Extracellular histone $\mathrm{H} 1$ is neurotoxic and drives a pro-inflammatory response in microglia. [Internet]. F1000Research 2013; 2: 148.Available from: http://f1000research.com/articles/2-148\#.Uy35nyczAIM.mendeley

Haeusler AR, Donnelly CJ, Periz G, Simko E a J, Shaw PG, Kim M-S, et al. C9orf72 nucleotide repeat structures initiate molecular cascades of disease. [Internet]. Nature 2014; 507: 195-200.[cited 2014 Jul 10] Available from: http://www.ncbi.nlm.nih.gov/pubmed/24598541

$\mathrm{Hu}$ B-Y, Zhang S-C. Differentiation of spinal motor neurons from pluripotent human stem cells. [Internet]. Nat. Protoc. 2009; 4: 1295-304.[cited 2014 Jan 29] Available from:

http://www.pubmedcentral.nih.gov/articlerender.fcgi?artid=2789120\&tool=pmcentrez\& rendertype $=$ abstract

Jovic A, Mertens J, Boeynaems S. associated Modifiers of C9orf72 DPR toxicity implicate nucleocytoplasmic transport impairments in c9FTD / ALS [Internet]. Nat. Neurosci. 2015; 18: 1226-1229.[cited 2015 Aug 28] Available from: http://www.ncbi.nlm.nih.gov/pubmed/26308983

Kanno E, Ishibashi K, Kobayashi H, Matsui T, Ohbayashi N, Fukuda M. Comprehensive screening for novel rab-binding proteins by GST pull-down assay using 60 different mammalian Rabs. [Internet]. Traffic 2010; 11: 491-507.[cited 2014 Oct 20] Available from: http://www.ncbi.nlm.nih.gov/pubmed/20070612

Lee MCS, Miller E a., Goldberg J, Orci L, Schekman R. Bi-Directional Protein Transport Between the Er and Golgi. Annu. Rev. Cell Dev. Biol. 2004; 20: 87-123.

Levine TP, Daniels RD, Gatta AT, Wong LH, Hayes MJ. The product of C9orf72, a gene strongly implicated in neurodegeneration, is structurally related to DENN RabGEFs. [Internet]. Bioinformatics 2013; 29: 499-503.[cited 2013 Feb 27] Available from:

http://www.pubmedcentral.nih.gov/articlerender.fcgi?artid=3570213\&tool=pmcentrez\& rendertype $=$ abstract

MacLeod D, Rhinn H, Kuwahara T. RAB7L1 Interacts with LRRK2 to Modify Intraneuronal Protein Sorting and Parkinson's Disease Risk [Internet]. Neuron 2013; 77: 425-439.[cited 2015 Jan 11] Available from: http://www.sciencedirect.com/science/article/pii/S0896627312011208

Muresan V, Ladescu Muresan Z. Shared Molecular Mechanisms in Alzheimer's Disease and Amyotrophic Lateral Sclerosis: Neurofilament-Dependent Transport of sAPP, FUS, TDP-43 and SOD1, with Endoplasmic Reticulum-Like Tubules. [Internet]. Neurodegener. Dis. 2015; 16: 55-61.[cited 2015 Nov 26] Available from: http://www.ncbi.nlm.nih.gov/pubmed/26605911

Nordin JZ, Lee Y, Vader P, Mäger I, Johansson HJ, Heusermann W, et al. Ultrafiltration with size-exclusion liquid chromatography for high yield isolation of extracellular vesicles preserving intact biophysical and functional properties. [Internet]. Nanomedicine 2015[cited 2015 Feb 15] Available from: http://www.ncbi.nlm.nih.gov/pubmed/25659648 
Ostrowski M, Carmo NB, Krumeich S, Fanget I, Raposo G, Savina A, et al. Rab27a and Rab27b control different steps of the exosome secretion pathway. [Internet]. Nat. Cell Biol. 2010; 12: 19-30; sup pp 1-13.[cited 2014 Jan 24] Available from: http://www.ncbi.nlm.nih.gov/pubmed/19966785

Renton AE, Majounie E, Waite A, Simón-Sánchez J, Rollinson S, Gibbs JR, et al. A hexanucleotide repeat expansion in C9ORF72 is the cause of chromosome 9p21-linked ALS-FTD. [Internet]. Neuron 2011; 72: 257-68.[cited 2012 Nov 8] Available from: http://www.pubmedcentral.nih.gov/articlerender.fcgi? artid=3200438\&tool=pmcentrez\& rendertype $=$ abstract

Sellier C, Campanari M-L, Julie Corbier C, Gaucherot A, Kolb-Cheynel I, OuladAbdelghani M, et al. Loss of C9ORF72 impairs autophagy and synergizes with polyQ Ataxin-2 to induce motor neuron dysfunction and cell death. EMBO J. 2016; 35: 127697.[cited 2016 Dec 21] Available from: http://www.ncbi.nlm.nih.gov/pubmed/27103069

Spanò S, Liu X, Galán JE. Proteolytic targeting of Rab29 by an effector protein distinguishes the intracellular compartments of human-adapted and broad-host Salmonella. [Internet]. Proc. Natl. Acad. Sci. U. S. A. 2011; 108: 18418-23.Available from: http://www.pnas.org/content/108/45/18418.long

Stepto A, Gallo J-M, Shaw CE, Hirth F. Modelling C9ORF72 hexanucleotide repeat expansion in amyotrophic lateral sclerosis and frontotemporal dementia. [Internet]. Acta Neuropathol. 2014; 127: 377-89.[cited 2015 Jan 21] Available from: http://www.ncbi.nlm.nih.gov/pubmed/24366528

Takeuchi T, Suzuki M, Fujikake N, Popiel HA, Kikuchi H, Futaki S, et al. Intercellular chaperone transmission via exosomes contributes to maintenance of protein homeostasis at the organismal level [Internet]. Proc. Natl. Acad. Sci. 2015: 201412651.Available from: http://www.pnas.org/lookup/doi/10.1073/pnas.1412651112

Wang $\mathrm{X}$, Huang $\mathrm{T}, \mathrm{Bu} \mathrm{G}, \mathrm{Xu} \mathrm{H}$. Dysregulation of protein trafficking in neurodegeneration [Internet]. Mol Neurodegener 2014; 9: 31. Available from: http://www.ncbi.nlm.nih.gov/pubmed/25152012

Zhang K, Donnelly C, Haeusler A. The C9orf72 repeat expansion disrupts nucleocytoplasmic transport [Internet]. Nature 2015[cited 2015 Oct 3] Available from: http://www.ncbi.nlm.nih.gov/pubmed/26308891

Figure. 1. Compromised EV secretion and MVE formation in fibroblasts and iPSC-derived motor neurons from normal or C9ALS/FTD patients.

(a) NTA quantitative analysis of particle concentration $\left(\times 10^{8}\right)$ detected in conditioned serum-free medium after 16 hours culture from average of 4 control and 4 C9ALS/FTD patient derived fibroblasts. (b) Particle concentration in the above mentioned medium for each control and C9ALS/FTD patient cell line. (c) Semi-quantification of C9-3 fibroblasts derived exosomes evaluated by fluorescence-activated cell scanning (FACS) 
with CD9 and CD63. MFI, mean fluorescence intensity. (d) EEA1 and CD63 immunofluorescence on fibroblasts from N-2 and C9-1 patients. (e) Quantification of CD63 positive vesicles per cell in cells from (d and S2). Error bars represent SEM. (f) Concentration of particles detected by NTA in serum-free conditioned medium from 2 controls and 4 C9ALS/FTD patient iPSC-derived motor neurons. Cells were preincubated in the medium for 16 hours prior to collection. Asterisks denote one-way ANOVA test significant $\mathrm{P}$-value $\left({ }^{*} \mathrm{P}<0.05,{ }^{*} \mathrm{P}<0.01\right.$ and $\left.* * * \mathrm{P}<0.001\right)$.

Figure. 2. C9ORF72 interacts with RAB7L1 protein and mediate trans-Golgi network disturbance in C9ALS/FTD.

(a) Pull-down assay with 42 different glutathione S-transferase (GST)-tagged mammalian RAB proteins and Myc-tagged forms of human C9ORF72. (b) A wild-type, constitutively active GTP-bound RAB7L1 Q67L mutant (CA) and a constitutively negative GDP-bound RAB7L1 T21N mutant $(\mathrm{CN})$ with a FLAG tag were transiently expressed in C9ALS/FTD-patient fibroblast cells. The cells were examined for the expression of RAB7L1 and C9ORF72 fluorescence with a confocal fluorescence microscope. O/E: overexpression. (c) Duolink PLA in SH-SY5Y cells using antiC9ORF72 and anti-RAB7L1 antibodies with DAPI counterstain. Duolink PLA in SHSY5Y cells with single anti-C9ORF72 antibody and without primary antibodies are used as negative controls. (d) Co-immunoprecipitation of C9ORF72 and RAB7L1 mutant $(\mathrm{CA}$ or $\mathrm{CN})$. Agarose beads coupled with GFP-RAB (CA) and - RAB (CN) mutant were incubated with COS-7 cell lysates containing C9ORF72. (e) WB analysis C9ORF72 and VSP26 in iPSC-derived motor neurons from C9ALS/FTD patients. Representative C9ORF72, $\beta$-ACTIN and VPS26 bands. (f) Semi-quantitative analysis of C9ORF72 and VPS26 expression from (e). (g) Immunofluorescence for mannose 6- 
phosphate receptor (M6PR) in N4 and C9-1 patient fibroblast cells. (h) Quantification of M6PR positive area relative to nucleus area per cell in normal and C9ALS/FTD patient fibroblasts grown in normal medium (DMEM and 10\% FBS, fed). Error bars represent SEM. Asterisks denote one-way ANOVA test significant P-value $(* \mathrm{P}<0.05 ; * * \mathrm{P}<0.01$ and $* * * \mathrm{P}<0.001)$.

Figure. 3. Genetic ablation and over-expression of $R A B 7 L 1$ and $C 9 O R F 72$ recapitulates a C9ALS/FTD phenotype in SH-SY5Y cells and fibroblasts from C9ALS/FTD patients.

(a) NTA data on the total particle concentrations in serum-free conditioned medium of SH-SY5Y cells after RAB7L1 and C9ORF72 siRNA mediated knockdown. The data (n $=3$ ) are presented as mean \pm SEM. Asterisks denote one-way ANOVA test significant $\mathrm{P}$-value $(* \mathrm{P}<0.05$ and $* * * \mathrm{P}<0.001)$. (b) Representative WB picture of exosome-specific markers including ALIX and TSG101 in EVs from siRNA-treated SH-SY5Y cells. Semi-quantitative analysis of exosome surface antigens including CD9 (c), CD63 (d), and CD81 (e) using the exosome isolation and enrichment kits (JSR Life Sciences, Tsukuba, Japan). MFI, mean fluorescence intensity. The data $(\mathrm{n}=3)$ are presented as mean \pm SEM. Asterisks denote one-way ANOVA test significant $\mathrm{P}$-value $\left({ }^{*} \mathrm{P}<0.05\right.$ and **P $<0.01$ ). Quantification of CD63 positive vesicles per cell (f) and the total particle concentrations in serum-free conditioned medium in C9ALS/FTD fibroblast cells (g) are shown. Two days after a transfection with C9ORF72 and/or RAB7L1 plasmid, the vesicles and the total particle concentrations in serum-free conditioned medium are counted under a confocal fluorescence microscope or by NTA, respectively. The data (n $=3$ ) are presented as mean \pm SEM. Asterisks denote one-way ANOVA test significant P-value $(* \mathrm{P}<0.05$ and $* * \mathrm{P}<0.01)$. $\mathrm{O} / \mathrm{E}$ : overexpression. (h) Western blotting of 
C9ORF72 and RAB7L1 from cells transfected in (f) and (g), two days after a transfection with C9ORF72 and/or RAB7L1 plasmid.

\section{Figure. 4. Oligonucleotide-based C9orf72 knock down in C9ALS/FTD-patient} fibroblasts.

Locked nucleic acid (LNA) gapmer ASOs-based knock down in control (N2) (a) and C9ALS/FTD-patient fibroblasts (C9-2) (b). Cells were incubated for 48 hours with ASO1-1 and ASO2-1 and the knockdown efficiency (\%) of C9ORF72 expression levels are evaluated by qPCR. The data are presented as mean $\pm \mathrm{SEM}$. Relative quantification and Western blotting images of C9ORF72 in control fibroblasts (N2) (c) and in C9ALS/FTD-patient fibroblasts (C9-2) (d) 4 days after an ASO-based knock down. $\mathrm{n}=$ 3/group. The data are presented as mean \pm SEM. Asterisks denote one-way ANOVA test significant $\mathrm{P}$-value $(* \mathrm{P}<0.05$ and $* * \mathrm{P}<0.01)$. (e) The proposed mechanism of defective extracellular and intracellular vesicle trafficking due to C9ORF72 haploinsufficiency and a compromised C9ORF72- RAB7L1 pathway in C9ALS/FTD. 


\section{Figure 1}

a

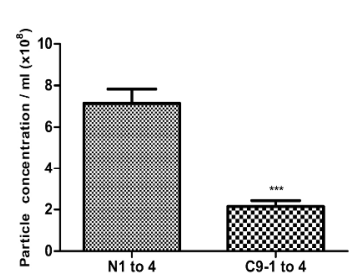

b

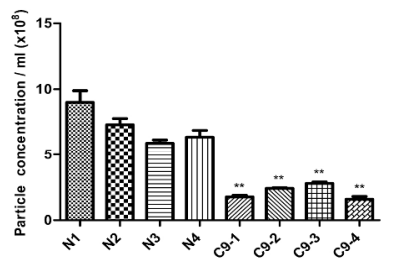

c

d
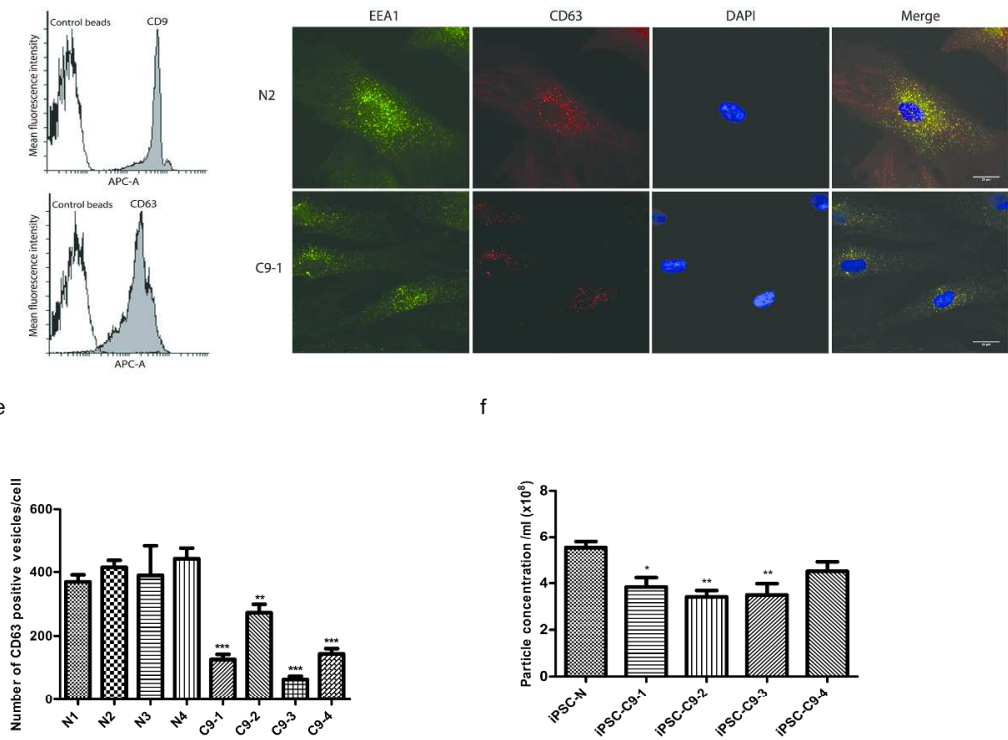

Figure 1

$216 \times 303 \mathrm{~mm}(300 \times 300$ DPI $)$ 
Figure 2
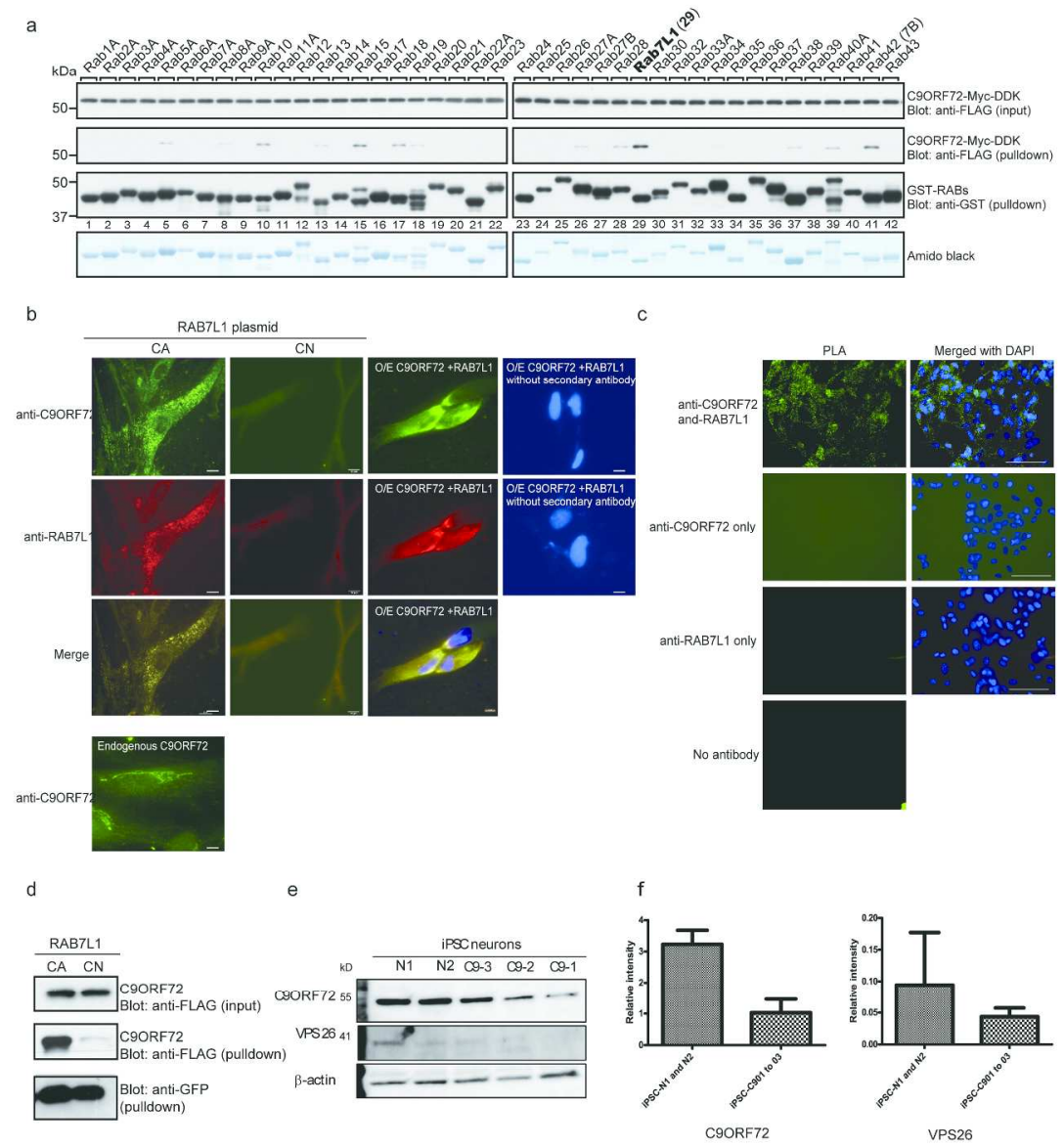

g
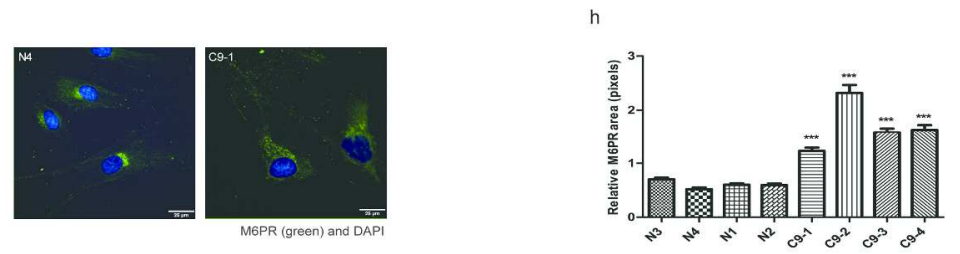

Figure 2

$216 \times 303 \mathrm{~mm}(300 \times 300$ DPI) 
Figure 3

a

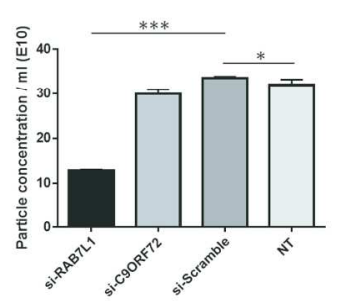

c

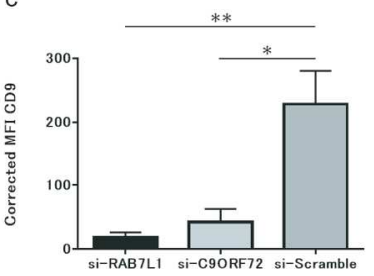

f

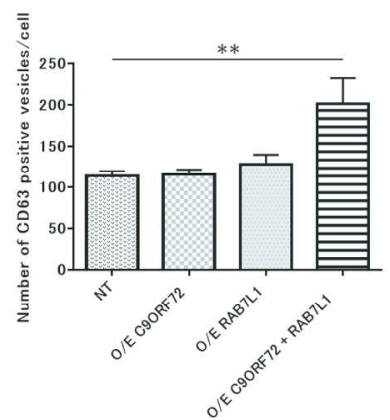

b
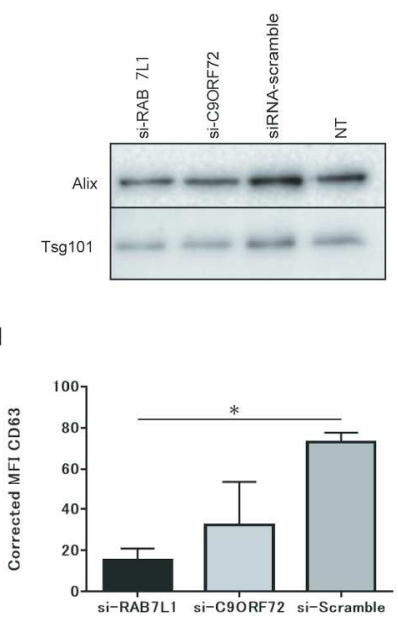

g
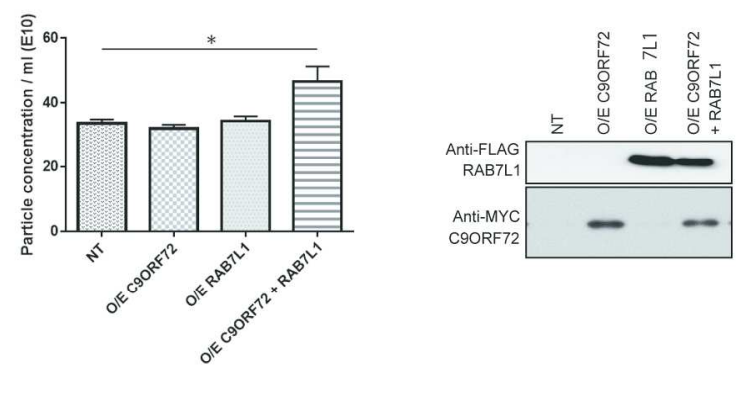

Figure 3

$203 \times 205 \mathrm{~mm}(300 \times 300 \mathrm{DPI})$ 
Figure 4

a

N2

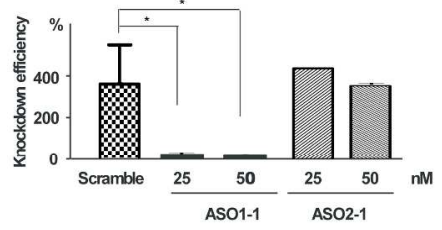

C $\quad$ 2
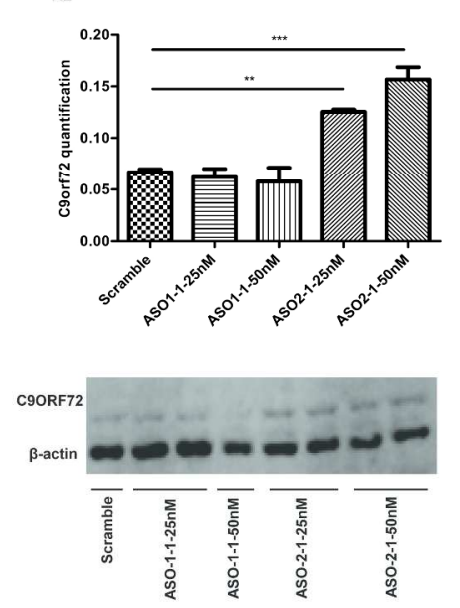

b

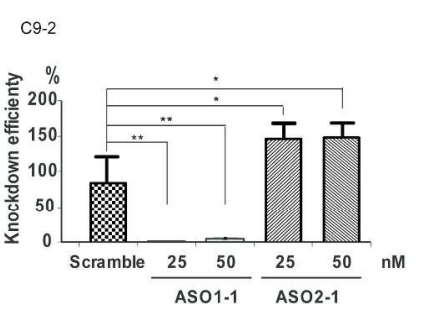

d C9-2
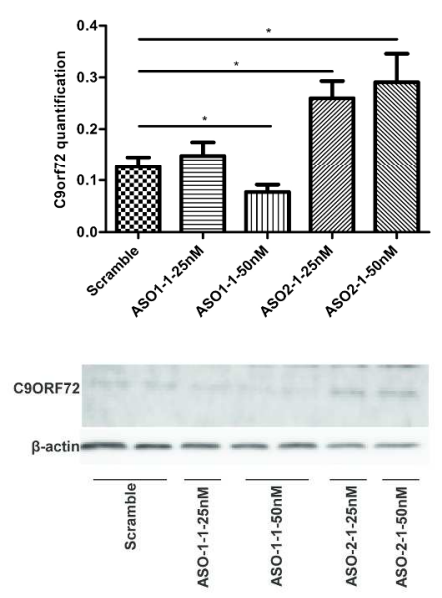

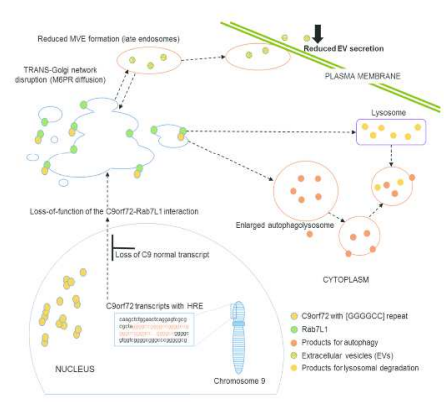

Figure 4

$210 \times 297 \mathrm{~mm}(300 \times 300 \mathrm{DPI})$ 
a

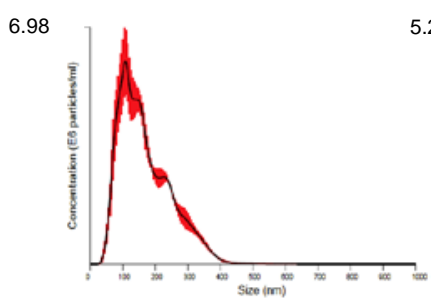

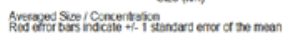

N1

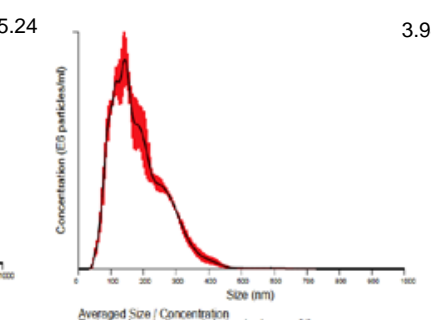

N2

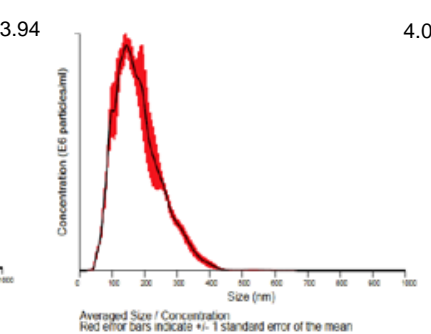

N3

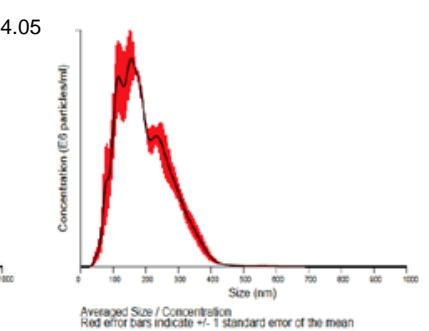

N4

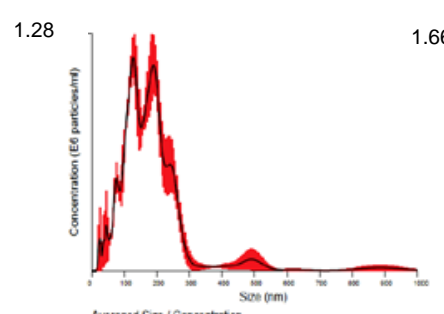

C9-1

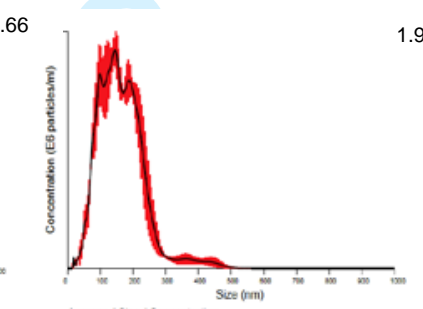

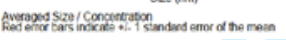

C9-2

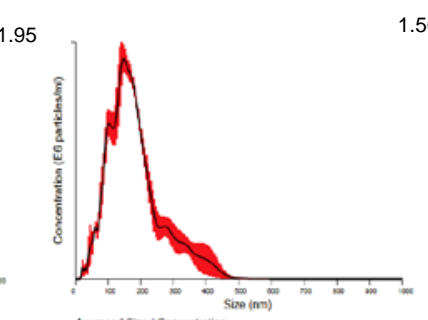

C9-3

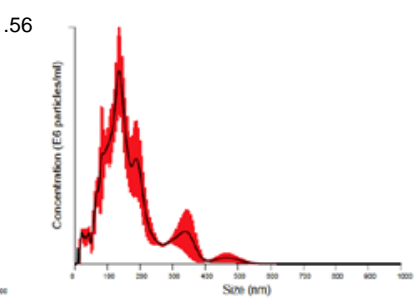

C9-4

Fig. S1. a) Size distribution profiles of particles by the nanoparticle tracking analysis detected in conditioned serum-free medium after 16 hours culture from 4 normal and 4 C9ALS/FTD-patient fibroblasts. 
b

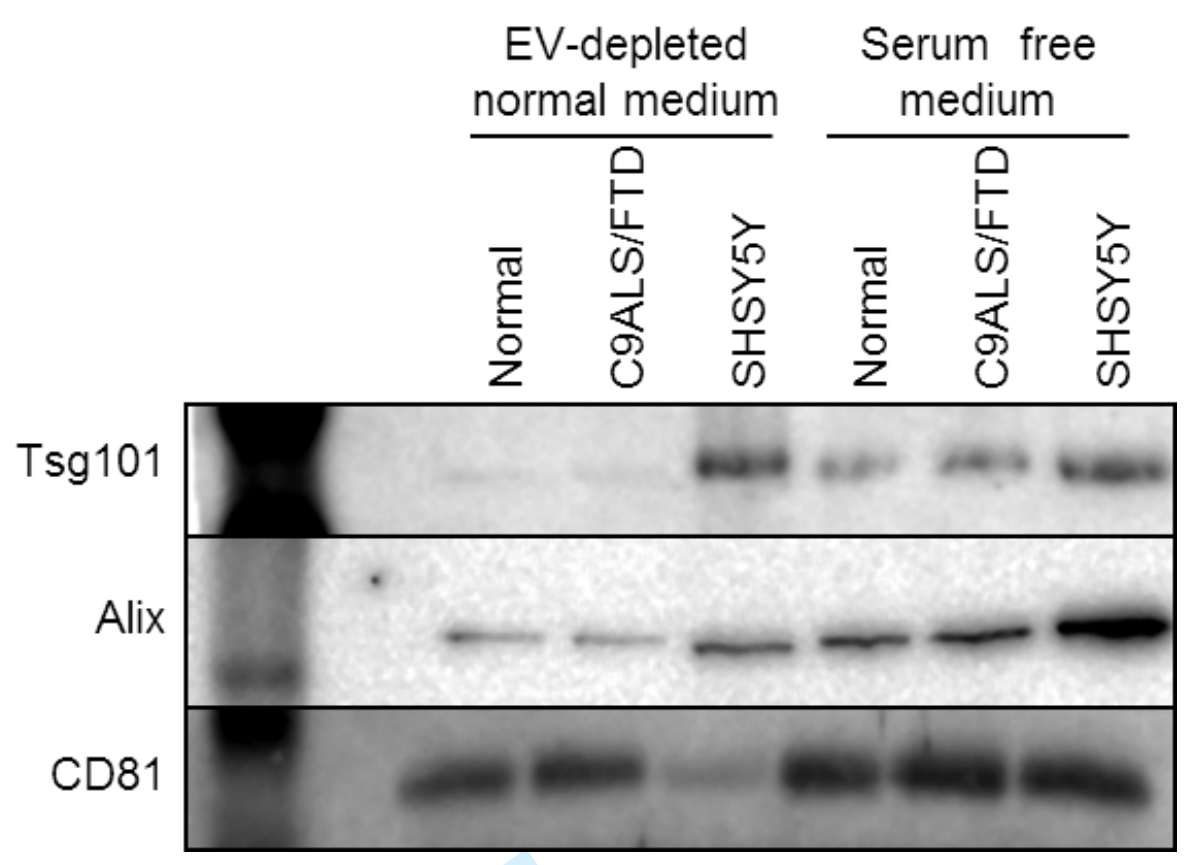

C

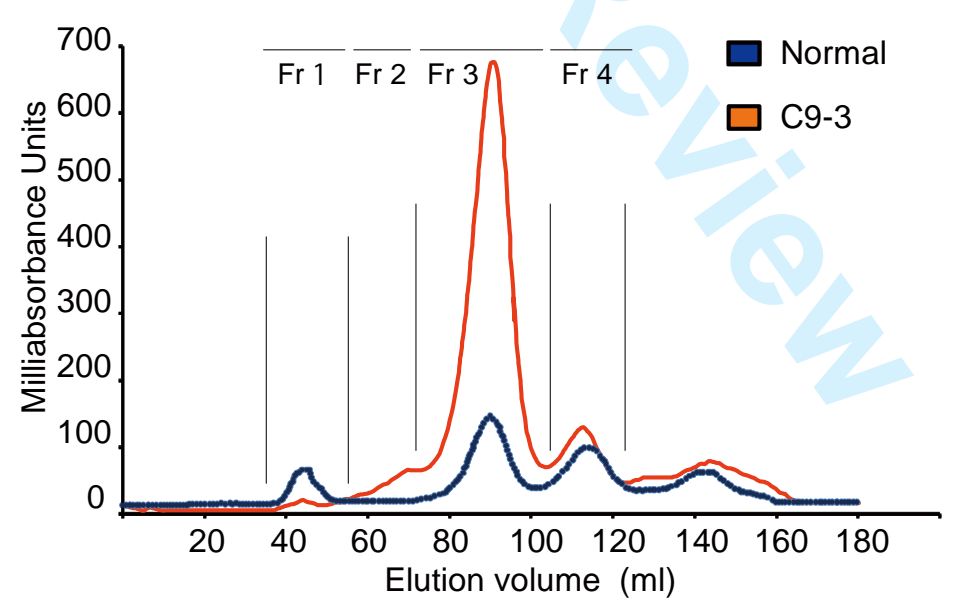

Fig. S1. Western blotting and HPLC in fibroblasts from control and C9ALS/FTD patients. b) Representative Western blotting of exosomal marker expression for TSG101, Alix and CD81 in control (normal) and C9ALS/FTD-patient fibroblasts. Human SH-SY5Y cells are used as a positive control. Cells were incubated in serum-free medium or EVs depleted normal medium with 10\% FBS for 48 hours. c) Liquid chromatography (LC) fractiona fraction. 


\section{Brain}

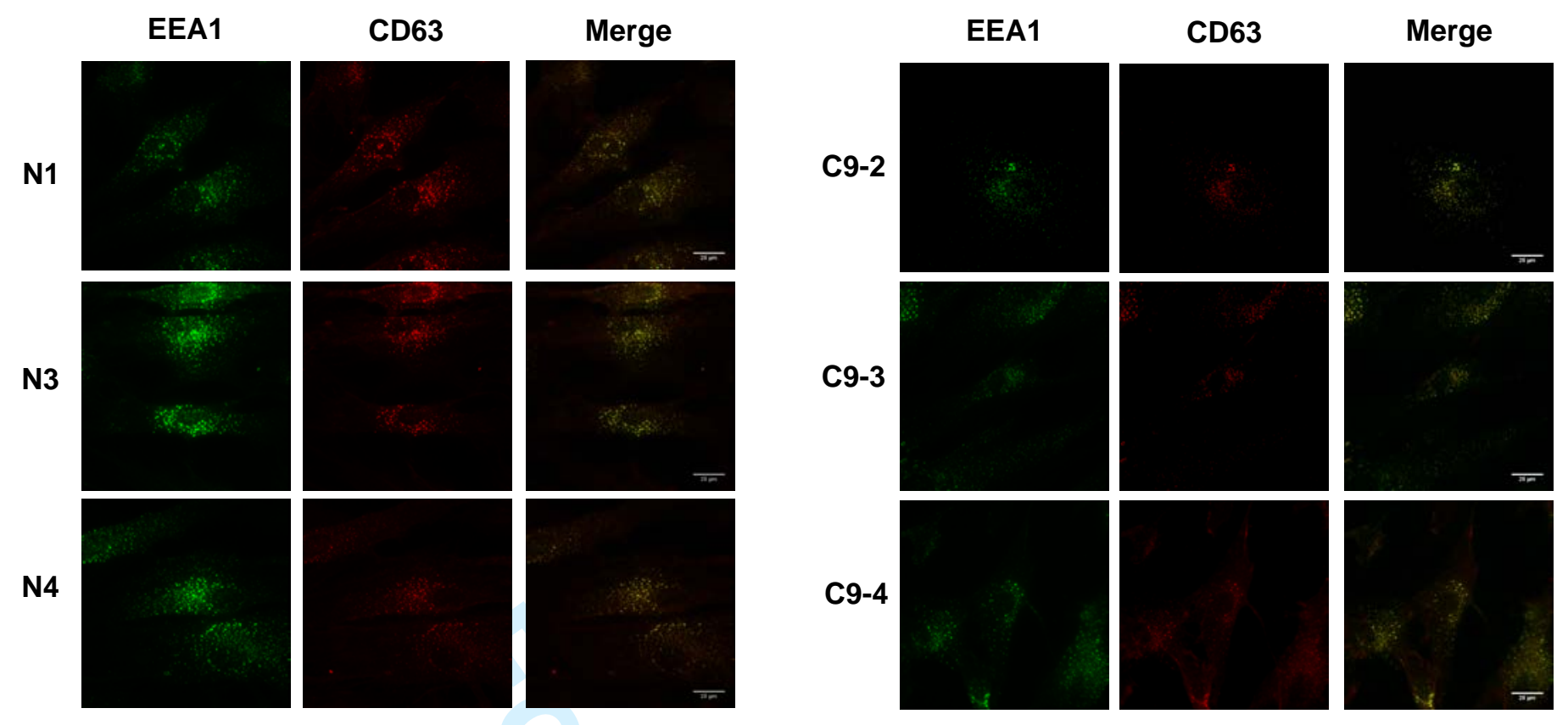

Fig. S2. Multivesicular endosome formation in fibroblasts from normal or C9ALS/FTD patients. EEA1 and CD63 immunofluorescence on fibroblast from normal and C9ALS/FTD patients. Bar $=25 \mu \mathrm{m}$. 
a
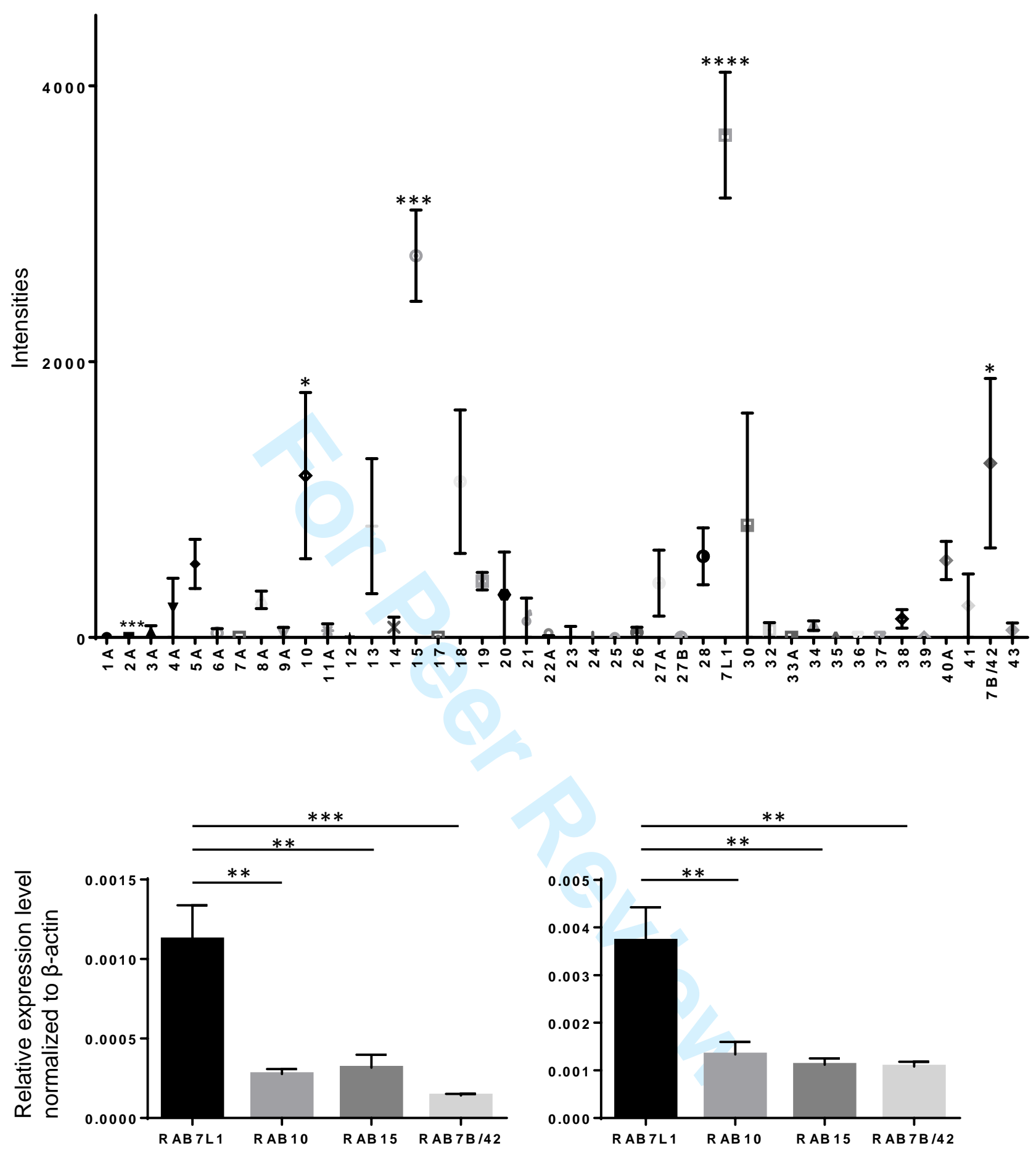

Fig. S3. C9ORF72 interacts with RAB7L1 to mediate trans-Golgi vesicle trafficking.

a) Quantitative analysis of pull-down assay with 42 different glutathione S-transferase-tagged mammalian RAB proteins and Myc-tagged forms of human C9ORF72. The intensities of bands on anti-flag Western blot measured by Image $\mathrm{J}$ are shown. The data $(n=3$, each) are presented as mean \pm SEM . Asterisks denote one-way ANOVA test significant $P$-value $\left({ }^{*} P<0.05\right.$; ${ }^{* *} P<0.01$; ${ }^{* \star *} \mathrm{P}<0.001$ and ; $\left.* * \star \mathrm{P}<0.0001\right)$. b) mRNA expression levels of RAB7L1, 10, 15 and7B/42 evaluated by qPCR assay in C9ALS/FTD-patient fibroblasts (upper panel) and -iPSC-neurons (lower panel) from C9ALS/FTD patients. Cells were incubated in DMEM with $10 \%$ fetal bovine serum. The data ( $\mathrm{n}=3$ / cell line) are shown as relative quantification to normal human fibroblast (N3, N4, C9-1, C9-2, C9-3) and iPSC-neurons (iPSC-N2, iPSC-N3, iPSC-C9-1, iPSC-C9-2, iPSCC9-3) presented as mean \pm SEM. *** $P<0.001$ 
Brain

GM130
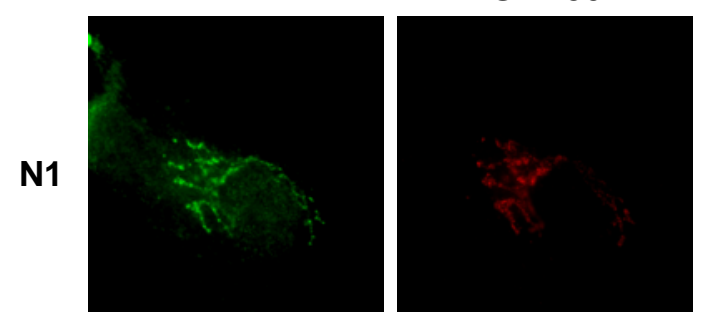

N2

N3

N4

C9-1
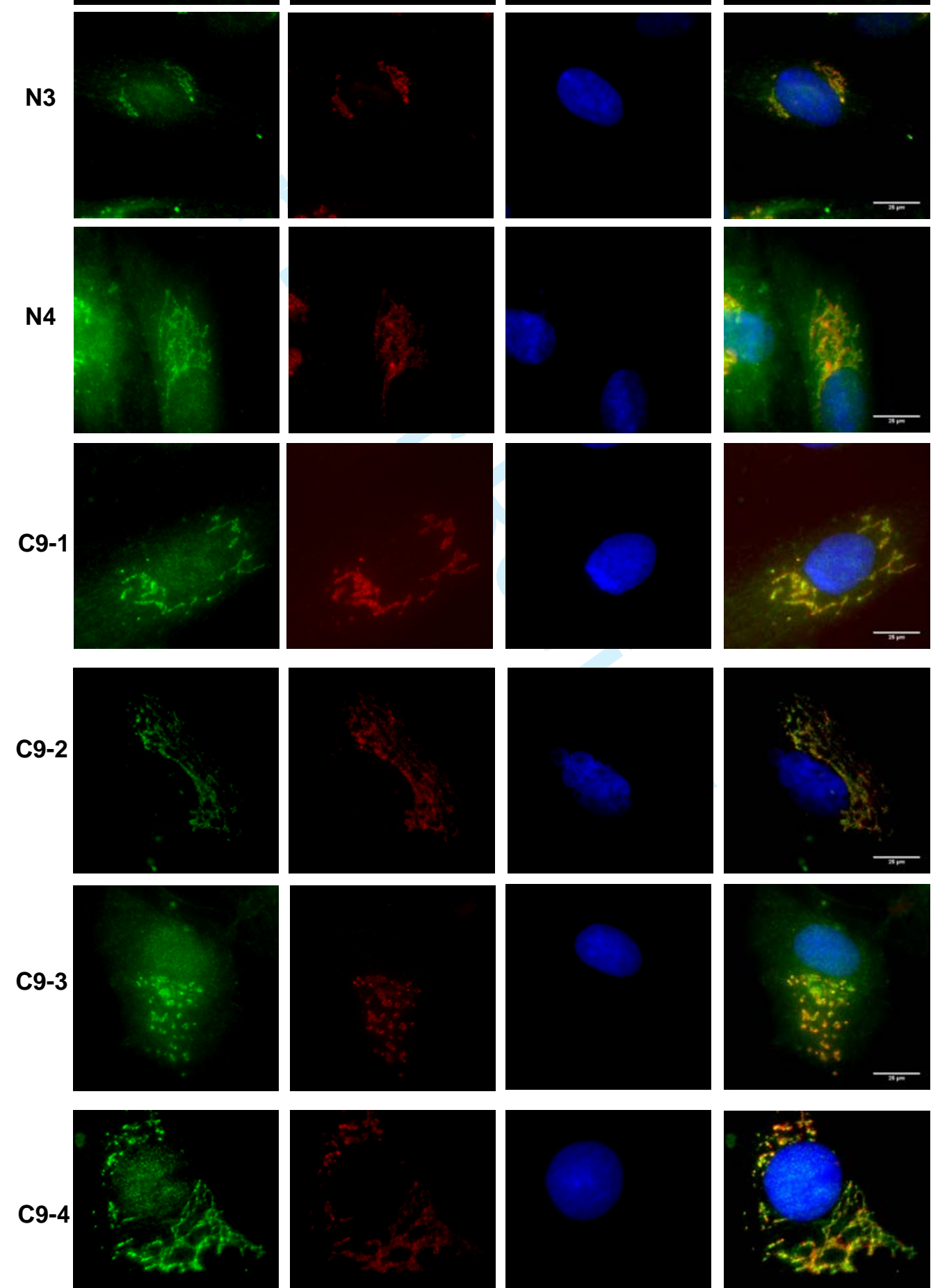

Fig. S4.
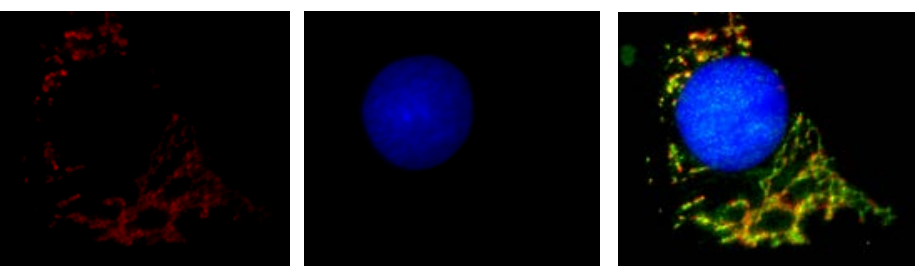
b
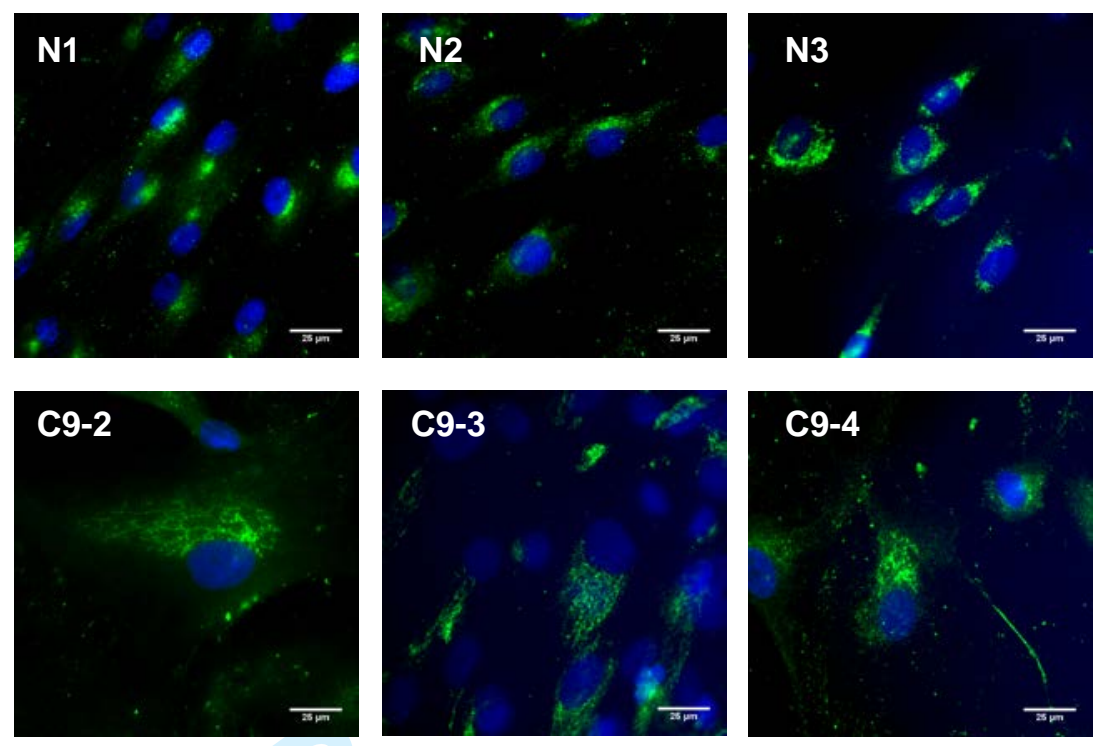

Fig. S4. C9ORF72 and M6PR are involved in extracellular vesicle and Golgi trafficking. (a) Immunofluorescence staining for C9ORF72 and the Golgi marker GM130 in fibroblasts derived from C9ALS/FTD and normal patients. Blue: DAPI. (b) Immunofluorescence image of M6PR in fibroblasts derived from C9ALS/FTD and normal patients. Blue: DAPI. Bar $=25$ $\mu \mathrm{m}$. 


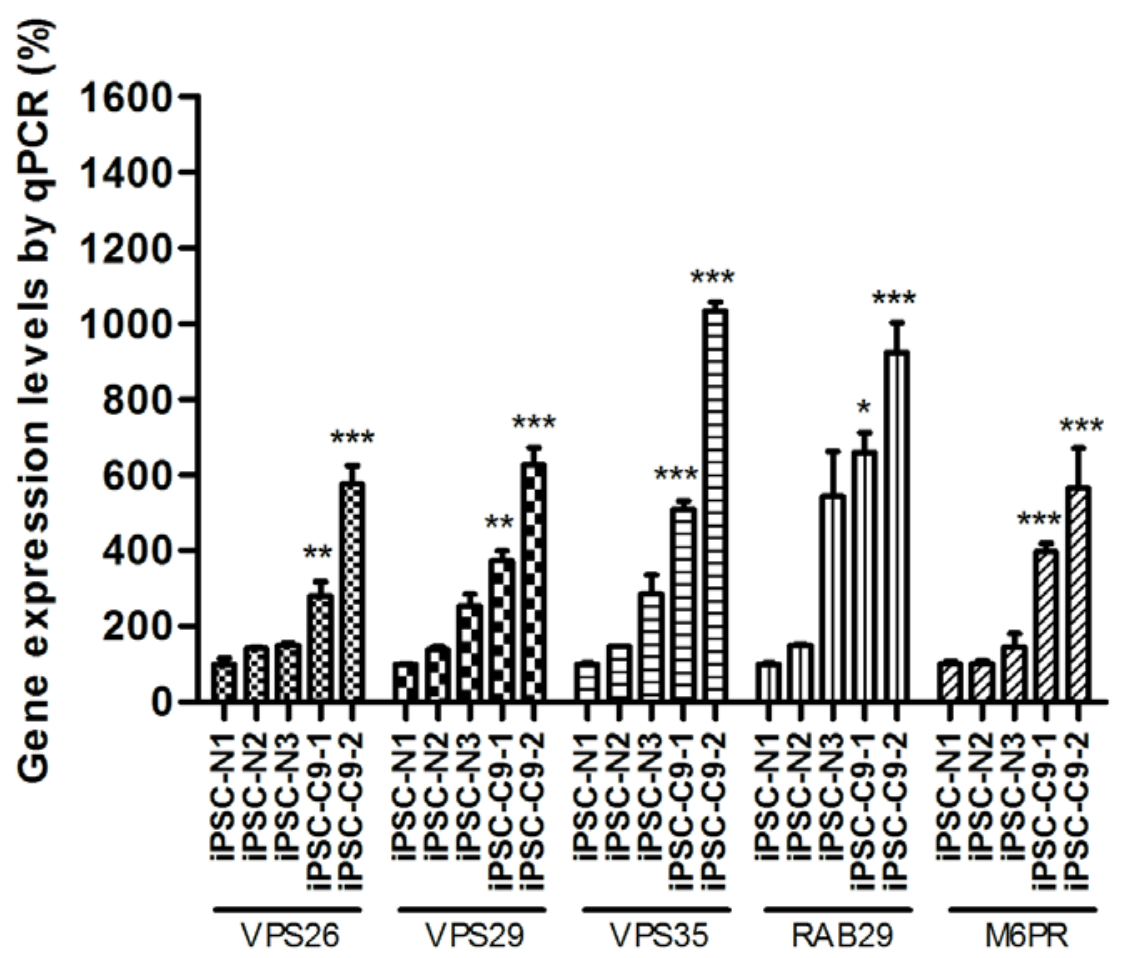

Fig. S5. C9ORF72 and RAB7L1 are involved in extracellular vesicle and Golgi trafficking. The gene expression levels of VPS26, VPS29, VPS35, RAB7L1 and M6PR measured by quantitative PCR in iPSC-neurons obtained from C9ALS/FTD patients (iPSCC9-1 and C9-2) and control (iPSC-N1 to N3). The data ( $n=3$, each) are presented as mean \pm SEM. Asterisks denote one-way ANOVA test significant $P$-value $\left({ }^{*} P<0.05\right.$; ${ }^{*} P<0.01$; $\star \star \star P<0.001)$. 


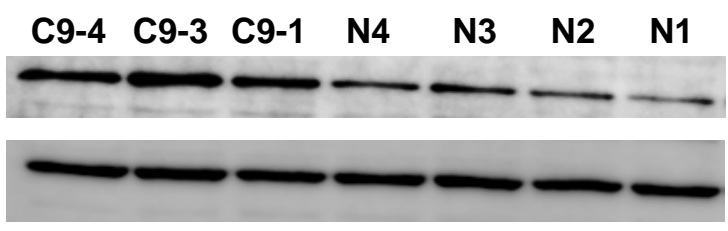

p62

$\beta$-actin

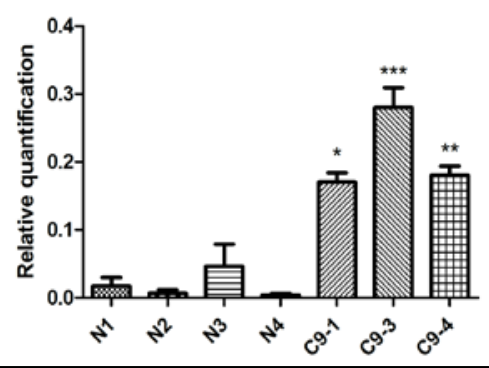

b

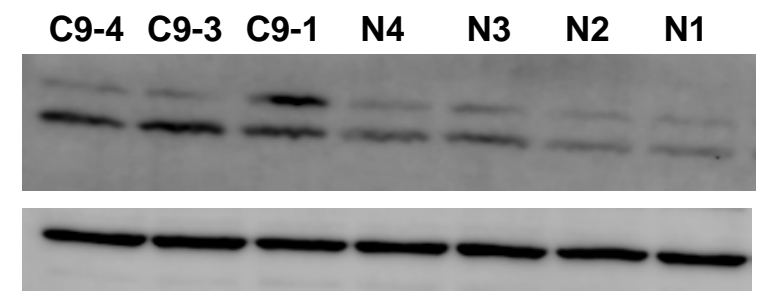

LC3-I

LC3-II

$\beta$-actin

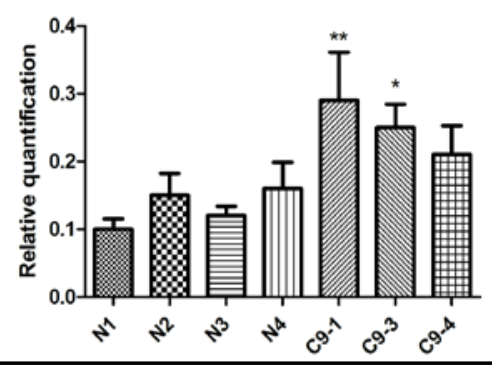

c

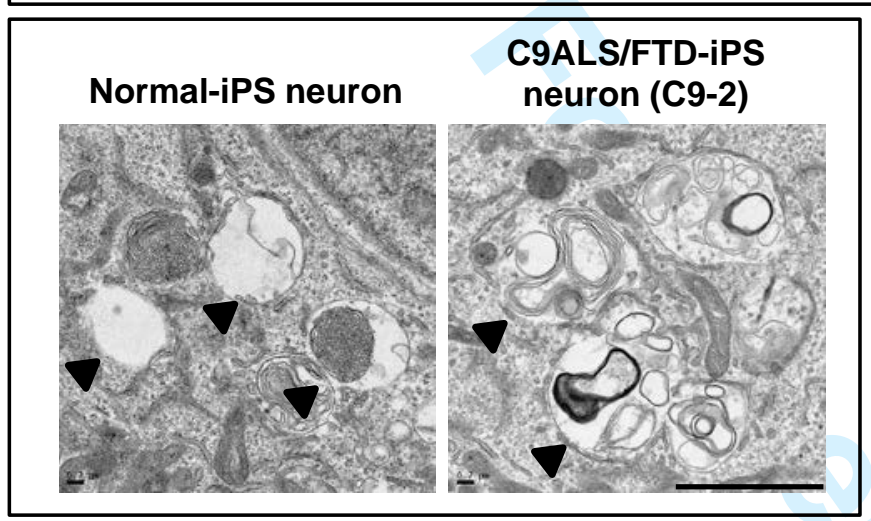

d

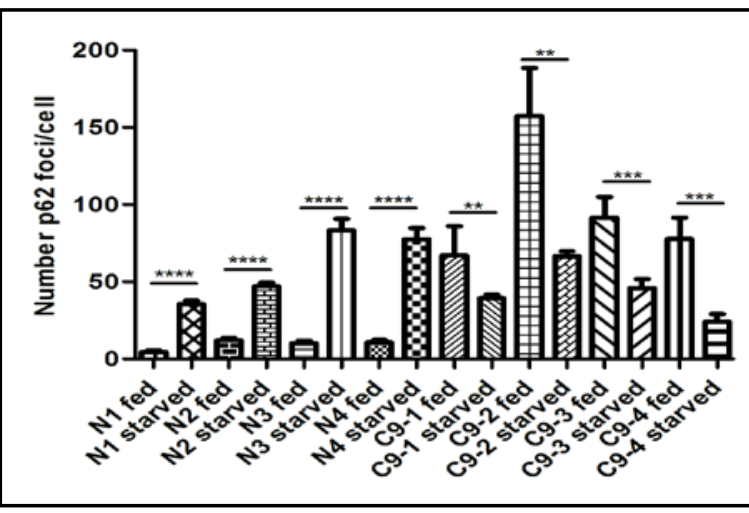

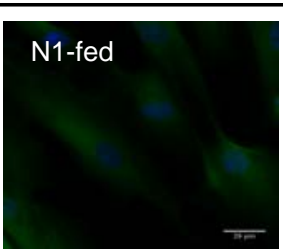
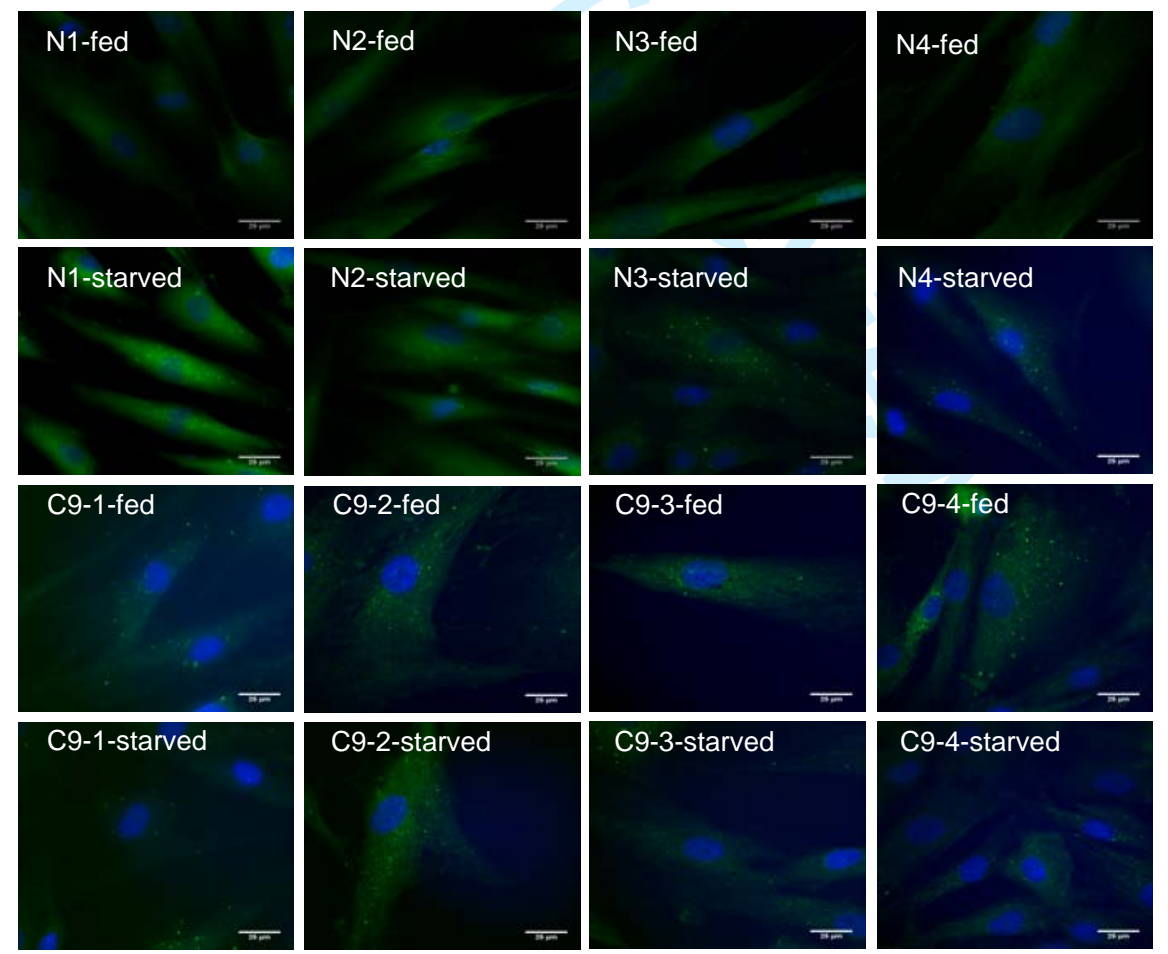

Fig. S6. Autophagy analysis in control and C9ALS/FTD patient derived fibroblasts and iPSC-derived neurons. p62 (a) and LC3-I and II (b) detection by Western blotting in protein lysates derived from four control (N1 to N4) and three C9ALS/FTD fibroblast cells (C9-1 to 3) grown in DMEM and 10\% FBS. Graphs show the comparison of the expression of p62 and LC3-II/I ratio between average of control lines and each C9ALS/FTD cell line. N=3/cell line. Asterisk denote oneway ANOVA test significant $P$-value $\left({ }^{*} P<0.05\right.$, ${ }^{*} P<0.01$ and $\left.{ }^{* * *} \mathrm{P}<0.001\right)$. The data are presented as mean $\pm S E M$. (c) Transmission electron microscope images of autophagosomes in normal (control) and patient (C9ALS/FTD) iPSC-neurons. Scale bar represents $0.7 \mu \mathrm{m}$. (d) Quantification of number of p62-positive autophagosomes by immunofluorescence in

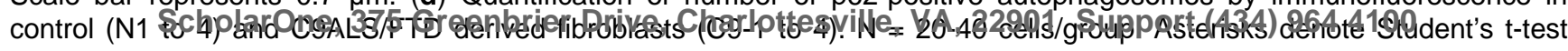
significant $P$-value $\left({ }^{*} P<0.05,{ }^{*} P<0.01,{ }^{* \star *} P<0.001\right.$ and $\left.{ }^{* \star *} \mathrm{P}<0.0001\right)$. (e) Representative images of cells analysed in (d). Scale bar represents $25 \mu \mathrm{m}$. 
a

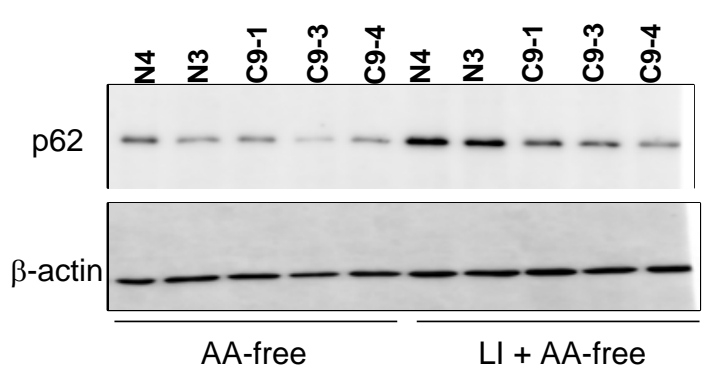

b

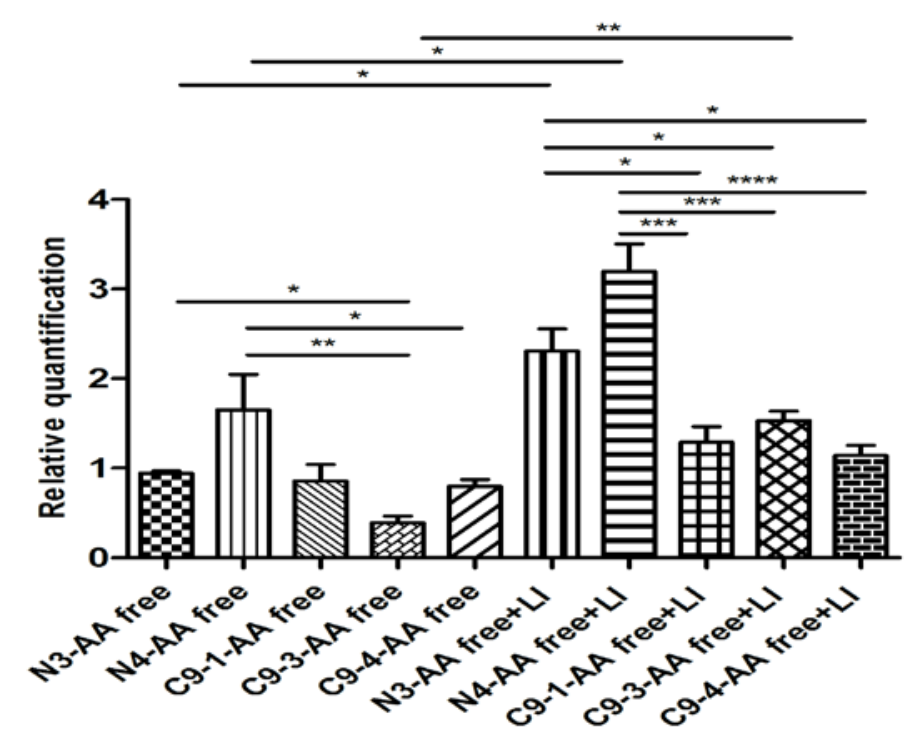

C
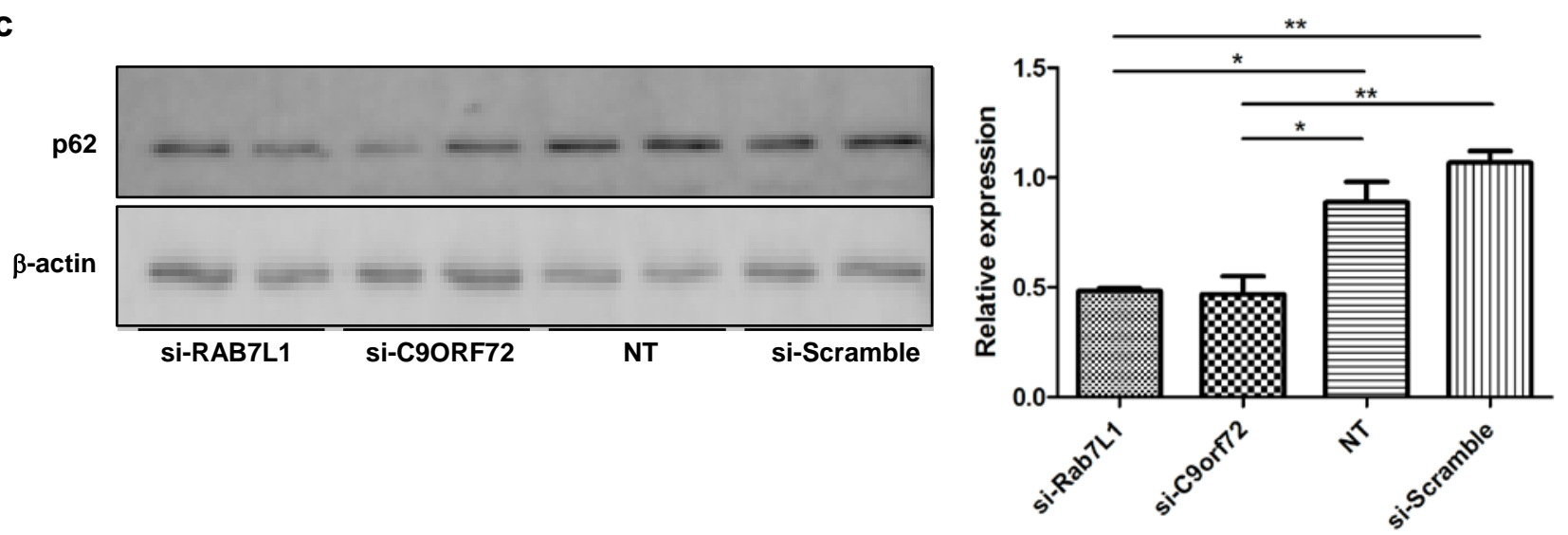

Fig. S7. Autophagy analysis in C9ALS/FTD patient fibroblasts and SH-SY5Y cells. p62 Western blotting (WB) image (a) and relative quantification (b) of protein lysates from control (N3 and N4) and C9ALS/FTD-derived fibroblast (C9-1 to C9-4) grown in normal medium (10\%FBS in DMEM), amino acid (AA)-free buffer (EBSS) and lysosomal inhibitor (LI) (E64d and pepstatin-A in EBSS for 4 hours. $\mathrm{N}=3$ /group. (c) p62 relative quantification WB in SH-SY5Y cell lysates after RAB7L1, C9ORF72 or scramble siRNA transfection. Cells were maintained in EBSS for 4 hours after 3 days transfection. NT, non-treated. Asterisk denote two-way ANOVA (for b) and one-way ANOVA test (for c) significant

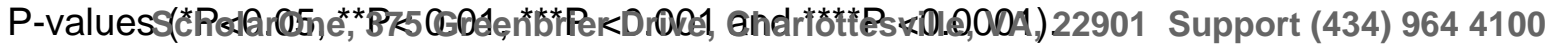



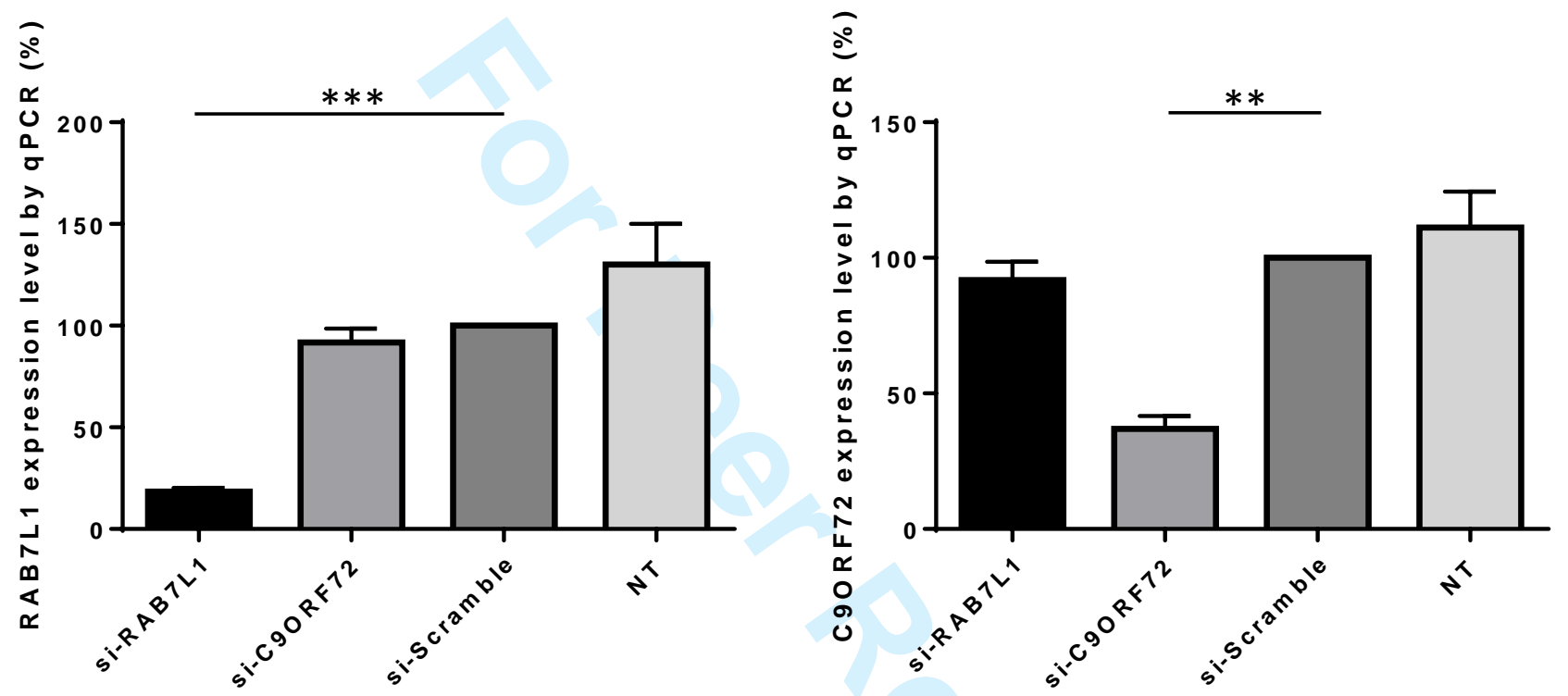

Fig. S8. RAB7L1 and C9ORF72 knockdown in SH-SY5Y cells. RAB7L1 and C9ORF72 gene expression levels measured by qPCR in SH-SY5Y cells after RAB7L1 or C9ORF72 si-RNA knockdown. NT, non-treated. The data $(n=3$, each) are presented as mean \pm SEM. Asterisks denote one-way ANOVA test significant $P$-value $\left({ }^{*} P<0.05\right.$; $\left.{ }^{* *} \mathrm{P}<0.01 ;{ }^{* \star *} \mathrm{P}<0.001\right)$. 


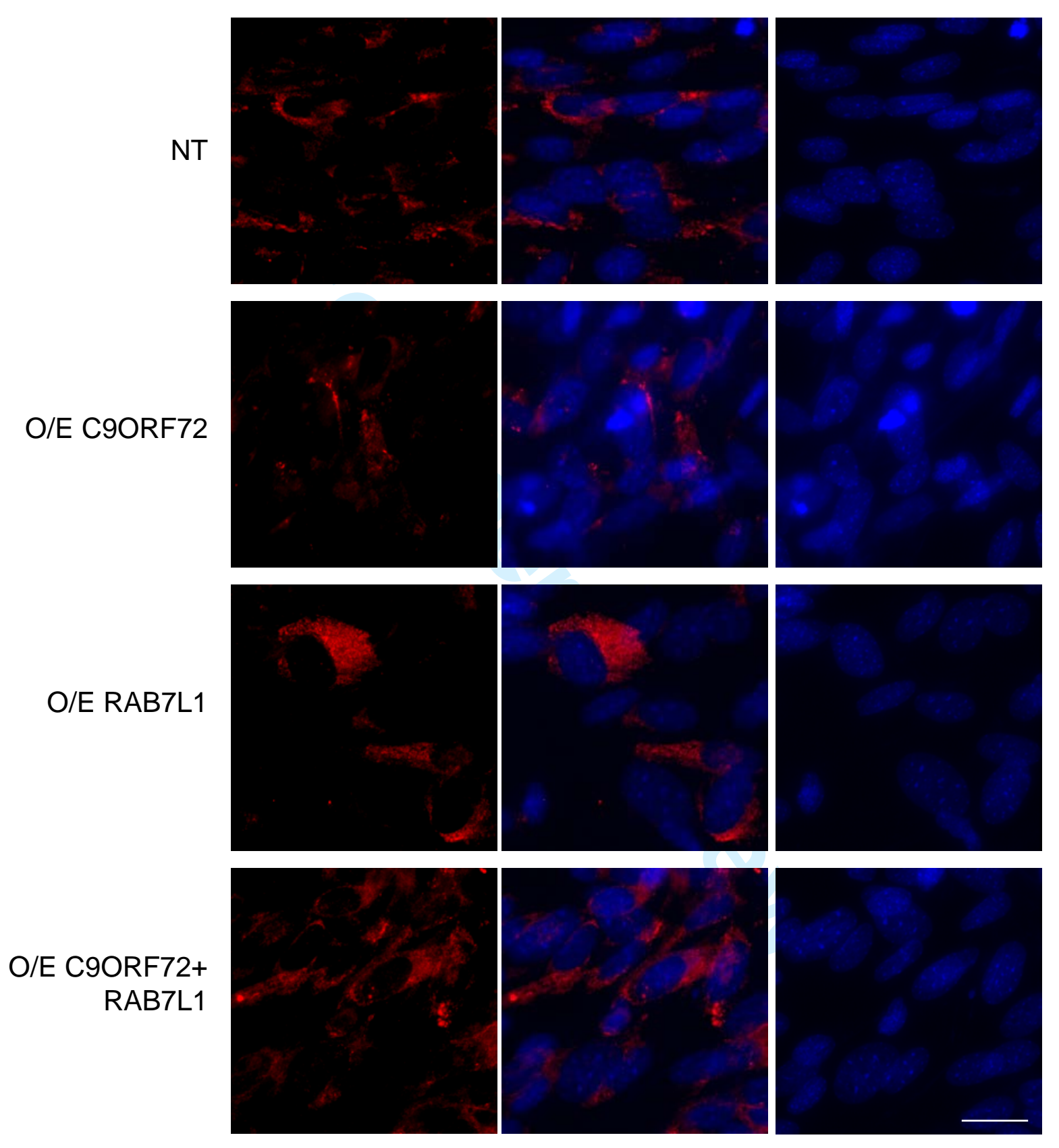

Fig. S9. CD63 immunofluorescence on SH-SY5Y cells two days after a transfection with C9ORF72 and/or RAB7L1 plasmid (4 $\mu$ g plasmid DNA). O/E: overexpression. Bar $=25$ $\mu \mathrm{m}$. NT, non-treated. 
a

RNA

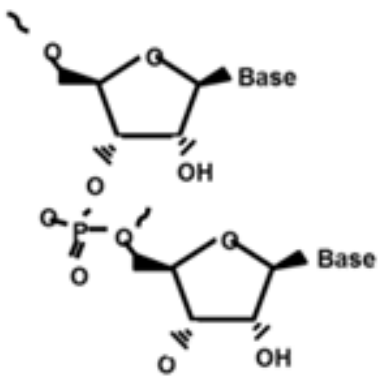

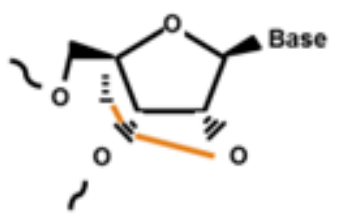

DNA-RNA hybrid

b

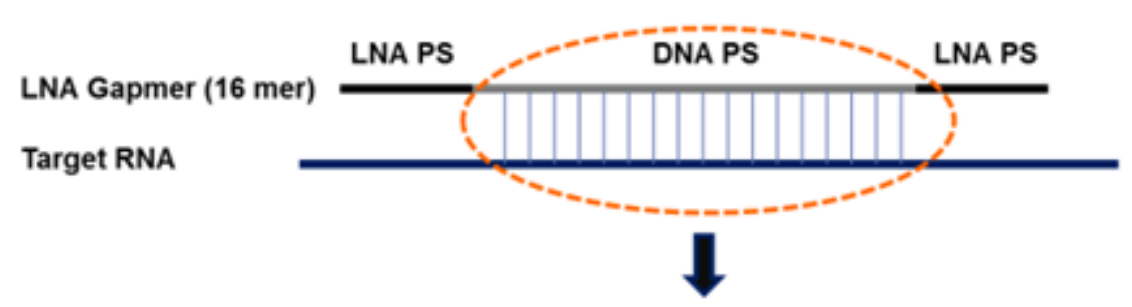

RNase H-mediated degradation of GGGGCC-repeat transcripts

c

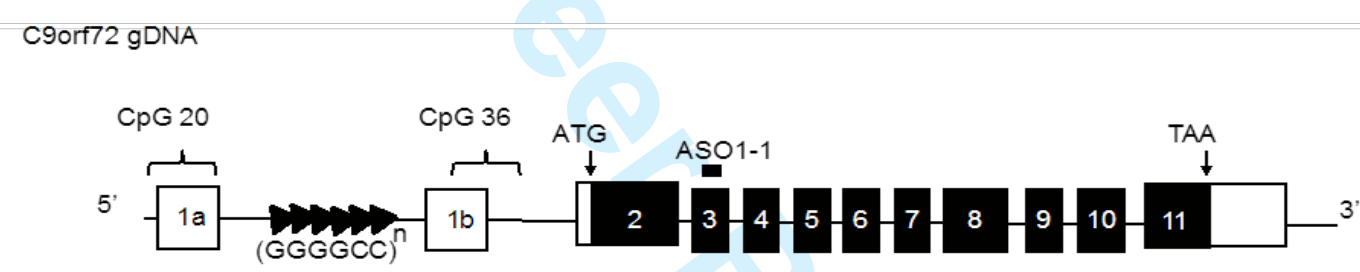

Transcripts

$1 b$

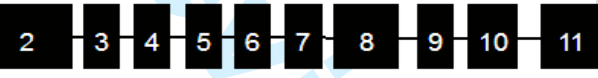

$\Delta 1 \mathrm{a}$
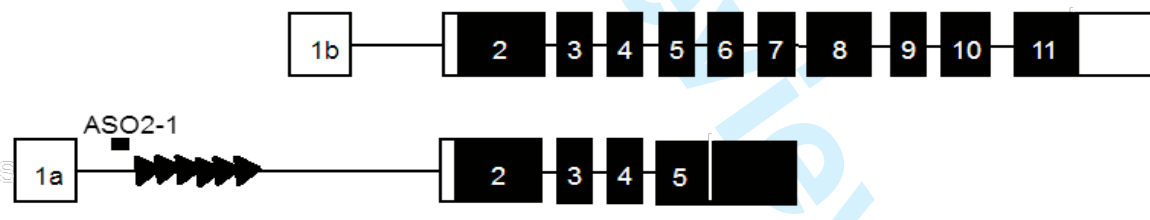

$2+3-4=5$

$1 \mathrm{a}$

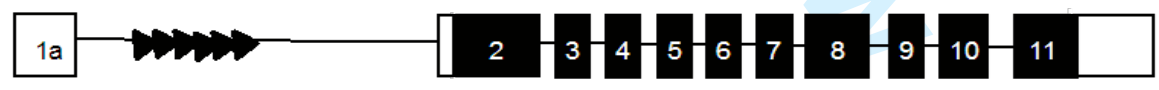

Fig. S10. LNA Gapmer oligonucleotide-based C9ORF72 knockdown strategy.

(a) Locked nucleic acid (LNA) is modified with an extra bridge (connecting the 2' oxygen and 4' carbon) and has high binding affinity to mRNA and high nuclease resistance. (b) Mechanism of LNA-based knockdown treatment. 'Gapmer' structures were developed where LNA phosphorothioate (PS) residues are present either side of DNA PS backbone. The external LNA PS residues thus enhance RNA binding while the internal DNA PS residues still allow RNase $H$ cleavage. (c) Graphical outline of the three possible C9ORF72 transcripts (1b, $\Delta 1 \mathrm{a}$ and $1 \mathrm{a})$ where the GGGGCC repeat expansion region lies between exons $1 \mathrm{a}$ and $1 \mathrm{~b}$. We designed two different antisense oligonucleotides (ASO1-1 and ASO2-1), targeting the C9ORF72 transcript. Black line indicates introns and squares indicate exons. 
a

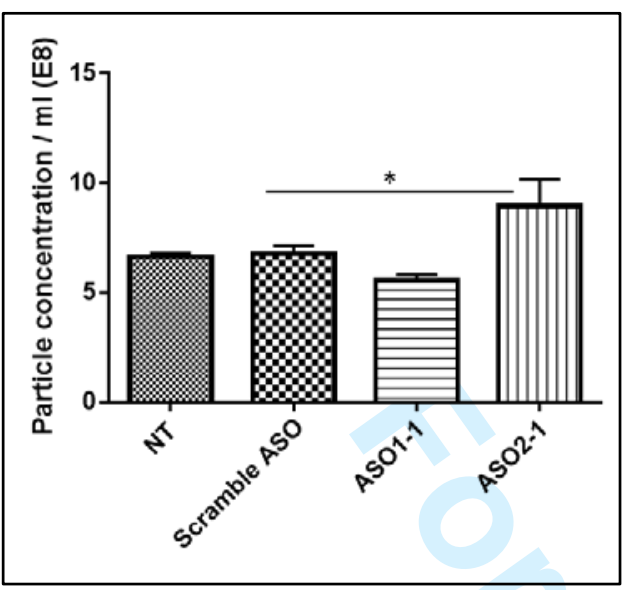

b

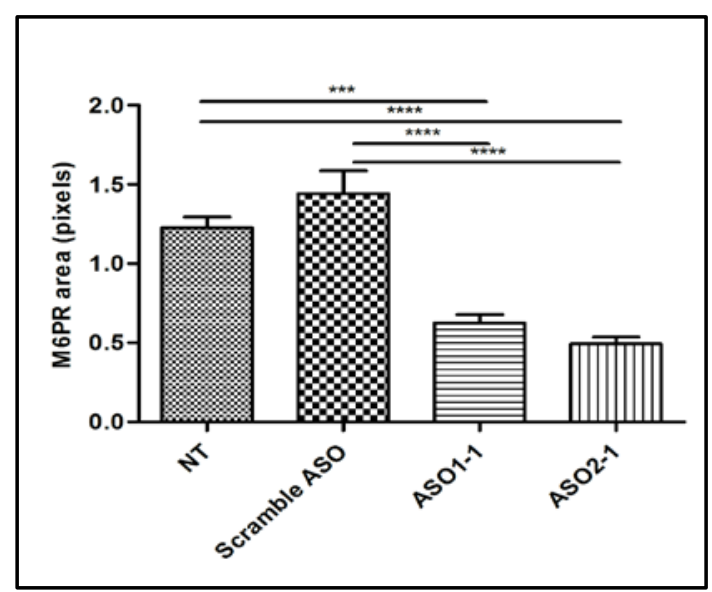

c
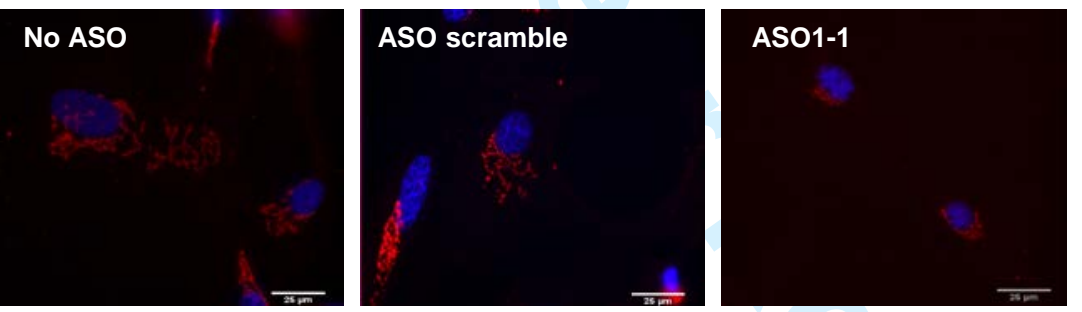

\section{ASO2-1}

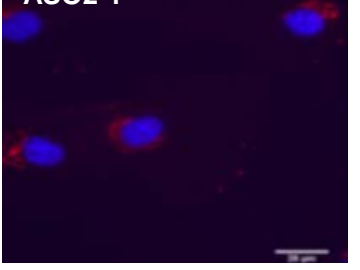

Fig. S11. Oligonucleotide-based C9ORF72 knock down in C9ALS/FTD patient fibroblasts normalizes EV trafficking and M6PR distribution.

(a) Nanoparticle Tracking Analysis (NTA) quantitative analysis of particle concentration ( $\left.\times 10^{8}\right)$ in the media from C9ALS/FTD fibroblasts treated with ASO1-1 and ASO2-1 and scramble LNA gapmers. (b) Quantification of M6PR positive pixels per cell in C9-3 fibroblasts treated with ASO1-1, ASO 2-1, scramble and non treated controls. Measurements were performed using Image J software plugin. $(n=25-50$ cells/group). Asterisks denote one-way ANOVA test significant $\mathrm{P}$-value $\left({ }^{*} \mathrm{P}<0.05,{ }^{* *} \mathrm{P}<0.01,{ }^{* * *} \mathrm{P}<0.001\right.$ and $\star \star \star \star * P 0.0001)$. Error bars represent \pm SEM. (c) Representative images of M6PR immunofluorescence from (b). Scale bar represents $25 \mu \mathrm{m}$. 


\section{Fibroblasts:}

N1: AGO2261

N2: AG05839

N3: NHDF

N4: N-N

C9-1: C9-T2

C9-2: C9-72

C9-3: C9-MS

C9-4: C9-DD

iPSC-derived neurons:

iPSC-N1: 180

iPSC-N2: 840

iPSC-N3: 841

iPSC-C9-1: C901-6

iPSC-C9-2: C902-2

iPSC-C9-3: C902-3

iPSC-C9-4: C904-1

Table S1. Nomenclature of the different patient fibroblast and iPSC neuron cell lines used in the present work.

\begin{tabular}{|c|c|}
\hline ASO 1-1 & $+C^{*}+A^{*}+C^{*} C^{*} A^{*} C^{*} T^{\star} C^{*} T^{\star} C^{*} T^{\star} G^{*} C^{*}+A^{*}+T^{*}+T$ \\
\hline ASO 2-1 & $+G^{*}+C^{*}+G^{*} A^{*} C^{*} T^{*} C^{*} C^{*} T^{*} G^{*} A^{*} G^{*} T^{*}+T^{*}+C^{*}+C$ \\
\hline ASO Scramble & $+C^{*}+A^{*}+G^{*} T^{\star} G^{*} T^{\star} G^{*} C^{*} T^{*} C^{*} A^{*} G^{*} T^{*}+C^{*}+A^{*}+A$ \\
\hline
\end{tabular}

Table S2. Sequence of the LNA Gapmer oligonucleotides (ASO) designed to knock down C9ORF72 transcripts.

+ , Locked nucleic acid (LNA), *, phosphorothioate linkage. 


\section{Inhibition of lysosomal enzymatic activity and autophagy flux measurement.}

Control and C9ALS/FTD patient-derived fibroblasts were grown in DMEM supplemented with 10\% FBS and $1 \%$ antibiotics in 6-well plates until reaching at least $80 \%$ confluence. For autophagy induction, medium was removed and cells washed with PBS $1 \mathrm{X}$ twice. Subsequently, cells were incubated in EBSS for 4 hours at $37^{\circ} \mathrm{C}$ and $5 \% \mathrm{CO}_{2}$. For inhibition of lysosomal enzymatic activity, EBSS was supplemented with E64d and Pepstatin (Sigma) for 4 hours. After this incubation, cells were washed with cold PBS $1 \mathrm{X}$ and lysed in $0.5 \mathrm{M} \mathrm{Hepes} \mathrm{pH} \mathrm{7.2,5} \mathrm{M} \mathrm{NaCl}, 1 \mathrm{M} \mathrm{MgCl}, 1 \%$ Triton $\mathrm{X}-100$ lysis buffer for protein extraction and WB analysis of autophagy markers.

\section{Detection of EVs by fluorescence-activated cell scanning (FACS).}

EVs were incubated with 50,000 anti-CD63-, anti-CD9 or anti-CD81 beads overnight at $4^{\circ} \mathrm{C}$ with horizontal shaking. Beads were washed with PBS containing $2 \%$ BSA using a magnet. Subsequently, anti-CD63-, anti-CD9 or anti-CD81 beads were incubated with anti-CD63 (BD Pharmingen, 561983, 1:200), anti-CD9 (Abcam, ab187776, 1:250) or antiCD81 (BD Pharmingen, 551112, 1:200) antibodies, respectively, or isotype-control antibodies, for $2 \mathrm{~h}$ at room temperature with horizontal shaking. Beads were washed with PBS containing $2 \%$ BSA and resuspended in PBS containing $0.25 \%$ BSA for analysis. MFI (mean fluoresence intensity) values were acquired on a FACS Canto (BD).

\section{Western blotting (WB).}

WB procedure was modified depending on the identity of the protein to be analyzed. For the quantification of exosomal-related proteins WB was performed using either the Mini-PROTEAN Tetra cell (Bio-Rad) or the iBlotsystem (Invitrogen, Life Technologies) according to the manufacturer's instructions. To cross-compare the yield of EVs, we proceeded to load equal volumes of the resuspended EVs pellet or filtrate on the gel. For the Bio-Rad system, EVs samples and 2X Laemmli sample buffer (Bio-Rad) containing 5\% $\beta$-mercaptoethanol were mixed at 1:1 ratio and heated at $100^{\circ} \mathrm{C}$ for $10 \mathrm{~min}$. Samples were then loaded in a $1.5 \mathrm{~mm}, 10 \%$ Tris/Glycine SDS-polyacrylamide gel and ran at $170 \mathrm{~V}$ for $60-70 \mathrm{~min}$ in running buffer, until the dye front reached the bottom of the tank. Proteins on the gel were then transferred to a polyvinylidine fluoride (PVDF) membrane (Millipore) at $100 \mathrm{~V}$ for $60-70 \mathrm{~min}$ in transfer buffer containing $20 \%$ methanol. Membranes were then incubated in blocking buffer (5\% fat-free milk in Tris buffer saline with $0.1 \%$ Tween-20 (TBS-T) for 60 min at room temperature (RT) with gentle shaking. For the iBlot $₫$ system, $30 \mu \mathrm{l}$ of sample was mixed with a sample buffer, containing $0.5 \mathrm{M}$ ditiothreitol (DTT), $0.4 \mathrm{M}$ sodium carbonate $\left(\mathrm{Na}_{2} \mathrm{CO}_{3}\right), 8 \%$ SDS and $10 \%$ glycerol, and heated at $65{ }^{\circ} \mathrm{C}$ for $5 \mathrm{~min}$. Samples were then loaded in a NuPAGE $®$ Novex® $4-12 \%$ BisTris Gel and ran at $120 \mathrm{~V}$ in running buffer until the dye front reached the bottom of the gel. The proteins on the gel were transferred to an iBlot nitrocellulose membrane (Invitrogen) for $7 \mathrm{~min}$ with the iBlot system. Membranes were stained with Ponceau S dye that was later washed away with PBS before blocking with Odyssey blocking buffer for 60 min at RT with gentle shaking. After the blocking step, the membrane was incubated with freshly prepared primary antibody solution (mouse monoclonal anti-Alix [3A9], ab117600 and rabbit polyclonal anti-Tsg101, ab30871; both at 1:1,000 dilution from Abcam, Cambridge UK and rabbit polyclonal anti-CD81 (H-121), sc-9158 at 1:100 dilution (Santa Cruz, TX, USA) overnight at $4^{\circ} \mathrm{C}$ or 2 hours at RT. Membranes were washed three times, 10 min each using washing buffer (TBS-T) with vigorous shaking before adding the secondary antibody solution (goat anti-mouse IgG DyLight800 at 1:10,000 dilution if detecting Alix; goat anti-rabbit IgGDyLight-800 at 1:10,000 dilution for detecting CD9, CD81, Tsg101 and Calnexin) and incubated for 1 hour at RT. After the secondary antibody incubation, membranes were washed three times, 10 min each and visualised by scanning both 700- and 800-nm channels on a Licor Odyssey CLX infrared imaging system. For subsequent probing of other proteins on the same membrane, the membrane was washed three times, 10 min each before re-incubation with the next primary antibody.

For autophagy-related, VPS26 and C9orf72 protein analysis, cells were lysed in 0.1\% Triton X-100 containing buffer and stored at $-80^{\circ} \mathrm{C}$. Protein concentration was measured by the bicinchoninic acid assay method (Thermo Scientific, Pierce). $12 \mu \mathrm{g}$ of protein lysates were mixed to $6 \mathrm{X}$ Laemmli sample buffer and boiled for 5 minutes. Proteins were separated in 12\% SDS-PAGE gels under constant $100 \mathrm{~V}$ and electrotransferred to PVDF membranes (Amersham Hybond-P; RPN303F) for $1 \mathrm{~h}$ at $150 \mathrm{~mA}$. Subsequently, membranes were blocked in $1 \%$ (w/v) skimmed milk in TBS/T (25 mM Tris- $\mathrm{HCl}, \mathrm{pH} 7.4,137 \mathrm{mM} \mathrm{NaCl}, 2.7 \mathrm{mM} \mathrm{KCl}, 0.08 \%$ Tween-20) for $1 \mathrm{~h}$ at room temperature and incubated overnight in 1\% skimmed milk/TBS/T (rabbit polyclonal anti-VPS 26, GTX106297: rabbit polyclonal anti-LC3, MBL PM036 1:1000; rabbit polyclonal anti-p62, Abcam, ab91526 1:1000; rabbit polyclonal anti-C9orf72, Santa Cruz S-14 138763 1:1000, mouse monoclonal anti-beta-actin, Abcam, ab6276 1: 5000). After 3 washes with TBS/T buffer, membranes were incubated with fluorescence-conjugated secondary antibodies (IRDye 800CW goat anti-mouse IgG 1: 5000 and Licor IRDye 680RD goat anti-rabbit IgG 1:2000) diluted in 1\% skimmed milk/TBS/T and visualised in the Licor Odyssey CLx infrared imaging system.

\section{Transmission electron microscopy (TEM).}

A 200 mesh nickel carbon/formvar grid (Agar Scientific) was placed onto a $10 \mu \mathrm{l}$ droplet of the EVs re-suspension after UC for $15 \mathrm{~min}$. The grid was then blotted dry with filter paper, immediately transferred to a $15 \mu \mathrm{l}$ droplet of $2 \%$ uranyl acetate for $1 \mathrm{~min}$ and protected from light. The grid was again blotted dry with filter paper before being transferred to a $15 \mu \mathrm{l}$ droplet of filtered distilled and deionized water $\left(\mathrm{ddH}_{2} \mathrm{O}\right)$ for $1 \mathrm{~min}$. The grid was then blotted dry and left to air dry on the bench top for $15 \mathrm{~min}$. EVs negatively stained on this grid was then visualized with a JEOL 1010 transmission electron microscope (JEOI Tokvo, Japan) Drive, Charlottesville, VA, 22901 Support (434) 9644100 


\section{Co-localization analysis.}

C9ALS/FTD patient-derived fibroblasts were grown in coverslips under nutrient-rich (Dulbecco's modified Eagle's medium, supplemented with $10 \%$ foetal bovine serum) or starvation (EBSS serum-free medium for 4 hours) conditions. Briefly, cells were washed in PBS 1X, fixed in fresh 4\% paraformaldehyde in PBS for 10 minutes and permeabilized in $0.2 \%$ Triton X-100/PBS for an additional 10 minutes at room temperature. Subsequently, cells were blocked in 1\% bovine serum albumin (BSA)/PBS for 30 minutes and incubated with primary antibodies diluted in 1\% BSA/0.1\% Tween-20/PBS for 1 hour (goat anti-CD63, Santa Cruz Biotechnologies sc-31214 1:50; rabbit anti-p62, Abcam ab91526 1:250; mouse M6PR, Abcam ab2733 1:100; GM130, mouse Alexa 647 1:50; rabbit EEA1, Cell Signalling Technologies C45B10 1:100, rabbit anti-C9orf72, ThermoFisher Scientific PA5-31565, 1:25). After three washes in PBS, cells were incubated with secondary antibodies diluted in 1\% BSA/PBS for 1 hour (anti-mouse Alexa Fluor 488, anti-goat Alexa 647 and anti-rabbit Alexa 488 1:500). Finally, nuclei were counterstained with Hoechst and mounted in Dako fluorescent mounting medium.

Fluorescent microscopy was performed using a Zeiss LSM510 Meta confocal microscope. Images were taken at 40X magnification and analysed using NIH ImageJ software version 1.48. For M6PR quantification, the total area positive for M6PR staining was normalized to the nucleus area in each cell.

\section{Duolink In Situ PLA}

This experiment was basically performed along the manufacturer's instructions. Cells were fixed with 4\% PFA for 10 min and permeabilized in $0.5 \%$ Triton X-100 in PBS for 10 min. Cells were blocked with the blocking solution (Duolink II) for 30 min at $37^{\circ} \mathrm{C}$ and incubated with anti-mouse RAB7L1 (ab56443), and -rabbit C9ORF72 (sc-138763) primary antibodies (RAB7L1 plus C9ORF72) for $60 \mathrm{~min}$ at $37^{\circ} \mathrm{C}$. Cells were incubated with the two PLA probes (1:5) for 60 $\min$ at $37^{\circ} \mathrm{C}$ and then with the ligase at 1:40 in the solution for $30 \mathrm{~min}$ at $37^{\circ} \mathrm{C}$. Incubation with single anti-RAB7L1 or -C9ORF72 antibody was used as negative controls. Cells were incubated with the polymerase (1:80 in the solution) for $100 \mathrm{~min}$ at $37^{\circ} \mathrm{C}$. The samples were mounted with DAPI and analyzed in a fluorescence microscope.

\section{Co-immunoprecipitation}

pEF-BOS/C9-MycDDK (EF promoter) ${ }^{1}$, pEF-BOS/RAB7L1-FLAG (EF promoter) ${ }^{2}$, pEGFP-C1/RAB7L1 Q67L (CMV promoter $)^{3}$ and pEGFP-C1/RAB7L1 T21N (CMV promoter) ${ }^{4}$ vectors were used for this experiment. Western blot to estimate the expression of the vectors in COS7 cells was performed with Anti-FLAG antibody-HRP (Sigma A8592) or Anti-GFP antibody-HRP-DirecT (MBL 598-7) antibodies.

C9-MycDDK expressing cell lysate $(\sim 1 \mathrm{~mL})$ and then $40 \mu \mathrm{l}$ of anti-FLAG-tag (M2) -conjugated agarose beads (Sigma A2220) were added in a $1.5 \mathrm{~mL}$ tube. The tubes were rotated at $4^{\circ} \mathrm{C}$ for $2 \mathrm{~h}$, centrifuged at $3 \mathrm{~K}$ for $1 \mathrm{~min}$ and then washed with $1 \mathrm{~mL}$ of wash buffer (50 mM HEPES-KOH, pH 7.2, $150 \mathrm{mM} \mathrm{NaCl}, 1 \mathrm{mM} \mathrm{MgCl}$, and 0.2\% Triton X-100 w/o protease inhibitors) three times. Anti-FLAG(M2)-conjugated agarose beads were added to EGFP-Rab7L1 (T22N) or EGFP-Rab7L1 (Q67L) expressing lysates. Samples were rotated at $4^{\circ} \mathrm{C}$ for $2-6 \mathrm{~h}$ and centrifuged at $3 \mathrm{~K}$ for $1 \mathrm{~min}$. Supernatant was used as input samples. After washing the beads, proteins bound to the beads were analysed by $10 \%$ SDS-PAGE followed by immunoblotting, using an anti-Myc-tagged antibody and a horseradish peroxidase-conjugated anti-GST antibody (Santa Cruz Biotechnology, Inc.). The immunoreactive bands were visualized using enhanced chemiluminescence (GE Healthcare Ltd.).

\section{Supplementary references}

1. Fukuda, M., Aruga, J., Niinobe, M., Aimoto, S. and Mikoshiba, K. (1994) Inositol-1,3,4,5-tetrakisphosphate binding to C2B domain of IP4BP/synaptotagmin II. J. Biol. Chem. 269, 29206-29211

2. Fukuda, M. (2003) Distinct Rab binding specificity of Rim1, Rim2, rabphilin, and Noc2: Identification of a critical determinant of Rab3A/Rab27A recognition by Rim2. J. Biol. Chem. 278, 15373-15380

3. Itoh, T., Satoh, M., Kanno, E. and Fukuda, M. (2006) Screening for target Rabs of TBC (Tre-2/Bub2/Cdc16) domain-containing proteins based on their Rab-binding activity. Genes Cells 11, 1023-1037

4. Tamura, K., Ohbayashi, N., Maruta, Y., Kanno, E., Itoh, T. and Fukuda, M. (2009) Varp is a novel Rab32/38binding protein that regulates Tyrp1 trafficking in melanocytes. Mol. Biol. Cell 20, 2900-2908 